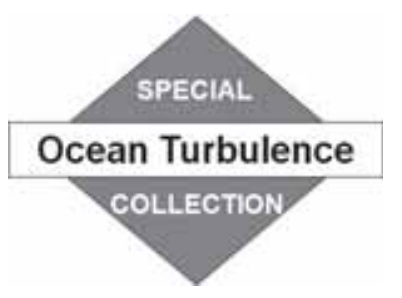

\title{
Adjustment of the Meridional Overturning Circulation and Its Dependence on Depth of Mixing
}

\author{
Catherine A. Vreugdenhil, Andrew McC. Hogg, Ross W. Griffiths, and Graham O. Hughes \\ Research School of Earth Sciences, Australian National University, Canberra, Australian Capital Territory, Australia
}

(Manuscript received 12 March 2015, in final form 9 July 2015)

\begin{abstract}
The relative roles of advective processes and mixing on the temporal adjustment of the meridional overturning circulation are examined, in particular the effects of mixing in either the abyssal or upper ocean. Laboratory experiments with convectively driven overturning and imposed stirring rates show that the circulation adjusts toward an equilibrium state on time scales governed by mixing in the upper boundary layer region but independent of the mixing rate in the bulk of the interior. The equilibrium state of the stratification is dependent only on the rate of mixing in the boundary layer. An idealized high-resolution ocean model shows adjustment (of a two-cell circulation) dominated primarily by the advective ventilation time scale, consistent with a view of the circulation determined by water mass transformation occurring primarily near the surface. Both the experiments and the model results indicate that adjustments of the circulation are controlled by surface buoyancy uptake (or rejection) and that the nonequilibrium circulation is dominated by advective processes, especially if the average abyssal ocean diffusivity is less than $3 \times 10^{-5} \mathrm{~m}^{2} \mathrm{~s}^{-1}$.
\end{abstract}

\section{Introduction}

The meridional overturning circulation (MOC) of the ocean involves the sinking of dense water at high latitudes in the North Atlantic and Southern Ocean. In the North Atlantic, the dense water sinking from the surface supplies an upper overturning cell in which the return upwelling is thought to occur primarily along isopycnals in the Southern Ocean, sloped due to Ekman pumping (Marshall and Speer 2012). The dense waters formed on the Antarctic shelves supply a bottom water (or lower) overturning cell in which the water is thought to upwell across isopycnals in the interior through a depth interval of order $2000 \mathrm{~m}$ before flowing back to the Southern Ocean along sloped isopycnals and reaching the surface at $65^{\circ}$ to $75^{\circ} \mathrm{S}$ (Marshall and Speer 2012). The meridional overturning circulation is an important contributor to the climate cycle via the uptake, storage, and transport of heat across the globe. To understand how the ocean

Corresponding author address: Catherine A. Vreugdenhil, Research School of Earth Sciences, Australian National University, Mills Rd. Bldg. 61, Canberra, ACT 0200, Australia.

E-mail: catherine.vreugdenhil@anu.edu.au reacts to changes in climatic conditions such as the surface buoyancy forcing or wind stress, we need to recognize the time scales and mechanisms of adjustment that govern the transient meridional overturning circulation.

The transience of the overturning circulation has been considered in numerical model studies for a singlehemisphere abyssal ocean (Kawase 1987) and a twohemisphere model coupled with surface observations (Yang 1999). It was suggested that the equilibration initially involves the propagation of Kelvin and Rossby waves across the ocean domain, after which the dynamics of the circulation change little and the system continues to adjust to a long-term stratification and overturning rate. Kelvin and Rossby wave propagation was found to influence the equilibrating ocean on a range of time scales from months or years (Kawase 1987; Yang 1999) up to several hundreds of years (Huang et al. 2000). Johnson and Marshall (2002) considered the response to a change in deep-water formation at high latitudes using an idealized reduced-gravity model to represent the upper cell in a two-hemisphere domain. While their results were largely consistent with Kawase (1987), the equator tended to act as a barrier to the 
interhemispheric communication and response. Here, we are primarily interested in the long-term equilibration of the MOC and the processes that can influence this adjustment.

One potential candidate in setting the long-term adjustment is diapycnal mixing across density surfaces. It seems clear that diapycnal mixing may play an important role in the ocean to allow the gravitationally stable density stratification to coexist with the vertical overturning circulation; so, it stands to reason that an adjustment time scale based on diffusivity may be significant. Theoretical scalings for simple equilibrium state ocean models often balance the turbulent mixing across density surfaces with the upward advection of dense water (Robinson and Stommel 1959; Welander 1971), assuming a governing role for abyssal diffusivity in the overturning. A leading-order role for mixing has been proposed for the abyssal lower overturning cell (Nikurashin and Ferrari 2013), where a local, steadystate balance between diffusivity and upward advection is assumed, following Munk (1966) and Munk and Wunsch (1998). However, interior mixing may not be required to sustain the upper-ocean overturning circulation; the waters can ventilate in the Southern Ocean (Toggweiler and Samuels 1998) as part of an overturning limb that has been characterized as adiabatic, with water mass conversion occurring only near the surface.

The adjustment could also be dependent on the location of mixing: energy budget arguments identify the near surface as the important mixing region. In a steadystate ocean with a linear equation of state, the rate of energy lost to mixing must exactly match the input of available potential energy by boundary buoyancy fluxes (Hughes et al. 2009) and direct numerical simulations (DNS) of purely convective overturning show that essentially all the irreversible mixing occurs within a convectively unstable mixed layer that forms at the surface in areas of destabilizing buoyancy flux (Gayen et al. 2013). This near-surface mixing is overwhelmingly the dominant energy sink for the mechanical energy supplied by surface buoyancy forcing. Extrapolation of these results to the ocean suggests that the energy consumed by abyssal mixing is likely to be only a small proportion of the overall budget for mixing throughout the ocean volume. At face value these results may appear to be at odds with studies focusing on abyssal mixing.

Ocean model studies indicate that the oceanic overturning and poleward heat transport are sensitive to the specified diapycnal diffusivity when it is relatively strong compared to ocean estimates (Bryan 1987; de Verdière 1988; Zhang et al. 1999; Mignot et al. 2006; Nikurashin and Vallis 2011, 2012). Theory and corresponding numerical models have shown that in the strong diffusivity limit the equilibrium circulation is controlled by this diffusivity, while in the weak diffusivity limit the circulation is governed by wind stress (in particular the wind stress across the Southern Ocean; Nikurashin and Vallis 2011, 2012). These authors suggested that the ocean lies in the weakly diffusive regime.

The studies referred to above all use a uniform vertical diffusivity, or a fixed-depth dependence of diffusivity, and thus do not provide insight into the roles of mixing at different levels. Cummins et al. (1990) compared ocean model runs having uniform diffusivity to runs having diffusivity increasing with depth at a rate inversely proportional to the buoyancy frequency. The intensified abyssal diffusivity increased the abyssal ocean temperature and the strength of circulation; this is unsurprising as the abyssal diffusivity was over an order of magnitude larger than that at the surface and large enough to be in the diffusion-dominated regime of Nikurashin and Vallis (2011). In a closed basin, singlehemisphere ocean model, Samelson (1998) found that enhancing the vertical diffusivity at the sidewalls caused the stratification to form separate boundary layer and abyssal regions, qualitatively similar to the real ocean, whereas the separate circulations were not present when mixing was uniform across the domain.

Other studies have concluded that abyssal mixing has a lesser influence than mixing in the upper ocean. From an idealized model with differing prescribed diffusivities in a near-surface (thermocline) layer and the deep interior, Scott and Marotzke (2002) concluded that the MOC heat and mass transport were determined mainly by mixing through the thermocline, whereas abyssal mixing did not substantially influence the flow. Saenko and Merryfield (2005) conducted an extensive parameter study into abyssal diffusivity in ocean models and found that while abyssal diffusivity influenced the abyssal circulation and stratification, it did not impact the heat transfer through the circulation. Similar conclusions were reached in Ferrari and Ferreira (2011), where the heat transport through ocean models was observed to be independent of abyssal diffusivity and governed by wind stress, with a lesser influence of diapycnal mixing at high latitudes in the North Atlantic. In a series of numerical models ranging from a simple model to an eddy-resolving simulation, Wolfe and Cessi (2011) found that, along with wind stress across the Southern Ocean, the nearsurface diffusivity at outcropping isopycnals in the North Atlantic governed the overturning, with no influence of interior diffusivity. The importance of near-surface mixing has been similarly noted in global circulation models (Jayne 2009). 
Returning to the dynamics of the adjustment of the circulation, another factor that is likely to be important in the long-term equilibration is the time required to pass the ocean volume through the system, which is described by a purely advective ventilation time scale. Indeed, Marotzke and Klinger (2000) find that the ocean responds on a time scale that is governed by advection in the deep western boundary current. It is not clear what the relative roles of the advection and mixing are or to what extent these are coupled in governing adjustments of the overturning transport and stratification.

We examine the relative roles of diapycnal mixing in the abyssal and upper ocean during adjustments toward long-term equilibrium states and consider the time scales of adjustment using complementary insights from conceptually simple laboratory experiments and a complex numerical ocean model. Our focus on the longterm adjustment time scales follows previous results in a thermally driven circulation that shows different scaling of equilibration times for boundary conditions of imposed flux and of imposed surface buoyancy such that the two time scales can be substantially different (Griffiths et al. 2013). The results for the case of imposed buoyancy in that study were consistent with a time scale expressly dependent on the diffusivity in the system. However, as the diffusivity was uniform through the depth, the influence of the spatial distribution of mixing was not addressed, and the study could not be extrapolated to the ocean without more detailed ocean modeling.

First, we report the results of a laboratory experiment in which an overturning circulation is forced by salt and freshwater buoyancy fluxes at the surface, with an imposed buoyancy throughput. These experiments follow from those of Stewart et al. (2012) and are conceptually similar to those of Whitehead and Wang (2008), having diapycnal vertical diffusivities that are externally imposed by small-scale turbulence generated by mechanical stirring. These previous experiments showed an increased overturning rate for stronger mixing in the long-time equilibrium circulation, consistent with earlier predictions from scaling theory for uniform mixing throughout the depth (Hughes and Griffiths 2006). Here, we abruptly impose, or in some cases change, the turbulent diffusivity, which is either uniform or limited to an upper region encompassing the surface boundary layer, and observe the temporal adjustment of the stratification to long-term equilibrium states. We also report complementary results of high-resolution ocean model simulations, which enable an examination of the roles of mixing in a more realistic two-cell ocean circulation, including realistic wind stress and surface buoyancy, with a restoring boundary condition. Diapycnal

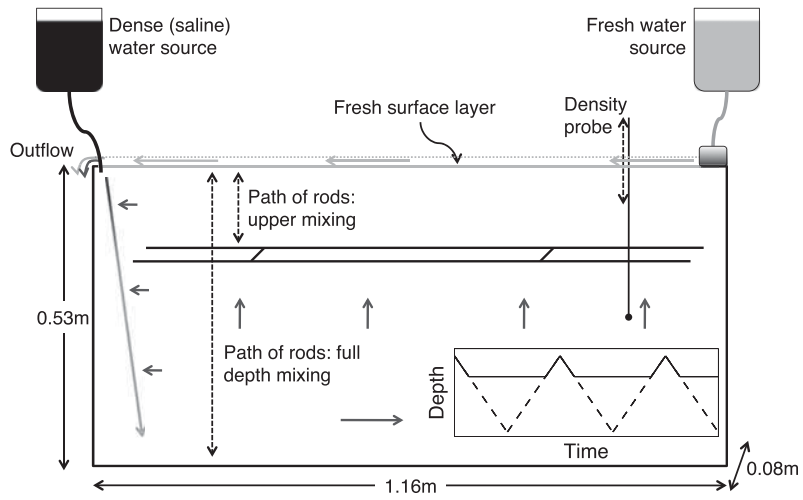

FIG. 1. The experimental arrangement. Below the saline source, the falling plume entrains interior water through the abyssal region. The freshwater source forms a surface layer that travels across the top of the working volume to the outflow. Inset indicates the mean path (without the superposed sinusoidal oscillations) of stirring rods with time; broken line is full-depth mixing, and solid line is upper mixing. Figure adapted from Stewart et al. (2012).

diffusivities are prescribed separately for the upper ocean and abyss, and the stratification and overturning transport are allowed to adjust to near-equilibrium states.

The laboratory experiment design, apparatus, and results are described in section 2 . The ocean model design and results are reported in section 3. In section 4, we discuss the dependence of the adjustment behavior on surface boundary conditions, and the different roles of advective and diffusive time scales, with concluding remarks in section 5 .

\section{Laboratory experiments}

\section{a. Experiment design and apparatus}

The apparatus and procedure are similar to that used in Stewart (2011) and Stewart et al. (2012). The working volume (pictured in Fig. 1) is a rectangular box tank of length $L=1.16 \mathrm{~m}$, width $W=0.08 \mathrm{~m}$, and depth $D=$ $0.53 \mathrm{~m}$, giving an aspect ratio of $D / L=0.457$. All experiments are carried out at room temperature, $21^{\circ} \pm$ $1^{\circ} \mathrm{C}$, and the tank is insulated with styrofoam on all sides, except the top, to reduce the impact of small fluctuations in room temperature. A vertical partition separates the main compartment (the working volume) from a thin compartment that continually drains to waste, effectively keeping the working volume constant throughout an experiment.

A saline source of density $\rho_{s} \approx 1022 \mathrm{~kg} \mathrm{~m}^{-3}$ is introduced at the top corner of the working volume (henceforth defined as the origin). The saline source is supplied from a 400-L reservoir through a constant head arrangement. The source tube is curled upward to 
TABLE 1. Experimental runs with the initial condition (initial), change in stirring, the applied diffusivity $\kappa$, the region over which stirring was applied, the exponential time scale $\tau$ for adjustment to equilibrium, the normalized value of $\tau$, the normalized interior density in the equilibrium state $\left(\rho_{f}-\rho_{0}\right) / \Delta \rho$, and the equilibrium boundary layer thickness $h$ (where $h$ contains $95 \%$ of the top-to-bottom density difference). Runs starting with a homogenous density tank (homog) are distinguished from those beginning with a fully convecting system (conv). The equilibrium convection in runs labeled with an " $a$ " formed the initial condition for the runs labeled with a " $b$ ". In run 3 , the initial density was larger than the final interior density; the adjustment was also slow enough that there was not enough saline water available in the reservoir to allow a second reequilibration. Uncertainties are $10 \%$ in $\kappa($ Stewart 2011$)$ and \pm 0.002 in $\left(\rho_{f}-\rho_{0}\right) / \Delta \rho$.

\begin{tabular}{|c|c|c|c|c|c|c|c|c|}
\hline Run & Initial & Change in stirring & $\kappa\left(\mathrm{m}^{2} \mathrm{~s}^{-1}\right)$ & Stirring & $\tau(\mathrm{h})$ & $\kappa \tau / h D$ & $\left(\rho_{f}-\rho_{0}\right) / \Delta \rho$ & $h(\mathrm{~mm})$ \\
\hline 1a & Homog & Apply & $5.59 \times 10^{-6}$ & Full depth & $2.0 \pm 0.3$ & $0.61 \pm 0.07$ & 0.279 & $124 \pm 6$ \\
\hline $1 \mathrm{~b}$ & Conv & Decrease & $4.76 \times 10^{-7}$ & Full depth & $5.7 \pm 0.9$ & $0.83 \pm 0.09$ & 0.743 & $22 \pm 1$ \\
\hline $2 \mathrm{a}$ & Homog & Apply & $4.76 \times 10^{-7}$ & Full depth & $6 \pm 2$ & $0.9 \pm 0.2$ & 0.664 & $22 \pm 3$ \\
\hline $2 b$ & Conv & Increase & $5.59 \times 10^{-6}$ & Full depth & $3.2 \pm 0.6$ & $1.0 \pm 0.1$ & 0.251 & $121 \pm 8$ \\
\hline 3 & Homog & Apply & $4.76 \times 10^{-7}$ & Full depth & $18 \pm 3$ & $0.91 \pm 0.09$ & 0.708 & $64 \pm 1$ \\
\hline $4 a$ & Homog & Apply & $2.02 \times 10^{-6}$ & Full depth & $2.1 \pm 0.4$ & $0.46 \pm 0.07$ & 0.388 & $61 \pm 7$ \\
\hline $4 b$ & Conv & Full to upper & $2.02 \times 10^{-6}$ & Upper & $4.9 \pm 0.8$ & $0.77 \pm 0.09$ & 0.419 & $86 \pm 4$ \\
\hline $5 \mathrm{a}$ & Homog & Apply & $5.59 \times 10^{-6}$ & Upper & $1.5 \pm 0.2$ & $0.44 \pm 0.05$ & 0.297 & $133 \pm 6$ \\
\hline $5 b$ & Conv & Decrease & $4.76 \times 10^{-7}$ & Upper & $6 \pm 1$ & $0.63 \pm 0.08$ & 0.733 & $28 \pm 2$ \\
\hline
\end{tabular}

form a jet of saline water angled toward a small lip extending horizontally from the top of the partition. This arrangement allows a turbulent endwall line plume to form (across the smallest dimension of the tank) with no vertical momentum at its source. From there the negatively buoyant plume sinks to the bottom of the tank. A freshwater inflow is placed at the other end of the tank to form a layer above the working volume, setting the upper surface boundary condition over the entire area at $\rho_{0} \approx 998.3 \mathrm{~kg} \mathrm{~m}^{-3}$. The effect of placing the saline source at the other end (beneath the freshwater input), thus reversing the direction of the large-scale circulation relative to the surface stress, was previously found to be negligible. The saline and freshwater inputs are kept at constant volume fluxes (per unit width) of $Q_{s}=$ $32.0 \mathrm{~mm}^{2} \mathrm{~s}^{-1}$ and $Q_{0}=148.8 \mathrm{~mm}^{2} \mathrm{~s}^{-1}$, respectively. These quantities fix the flux Rayleigh number $\mathrm{Ra}_{\mathrm{F}}=$ $B L^{3} / \kappa_{m}^{2} \nu_{m}=5.23 \times 10^{18}$, where $B=g^{\prime} Q_{s}$ is the imposed specific buoyancy flux, $g^{\prime}=g \Delta \rho / \rho_{0}$ is the reduced gravity, $\Delta \rho=\rho_{s}-\rho_{0}$ is the imposed density difference, $g$ is the gravitational acceleration, $\kappa_{m}\left(=1.39 \times 10^{-9} \mathrm{~m}^{2} \mathrm{~s}^{-1}\right)$ is the molecular diffusivity of salt in dilute aqueous solution, and $\nu_{m}\left(=1.15 \times 10^{-6} \mathrm{~m}^{2} \mathrm{~s}^{-1}\right)$ is the kinematic viscosity of the solution.

Mechanical stirring is introduced by two parallel 8-mm diameter stainless steel rods extending the length of the tank and positioned in a horizontal plane with their axes $32 \mathrm{~mm}$ apart. The rods are translated vertically through a predefined depth range with a sinusoidal oscillation superimposed on a constant translation speed. To relate rod speed to a turbulent vertical diffusivity $\kappa$, Stewart et al. (2012) considered the diffusion of dye tracer with each stirring rate. As the rods and their translation speeds and oscillation frequencies remain unchanged from this previous study, the same calibration is applied here to estimate vertical diffusivities. Two modes of operation are used for the mixing rates: the first has a depth range of the rods that is full depth; the second has a depth range through a shallow upper region of thickness $70-150 \mathrm{~mm}$ (chosen to be $30-40 \mathrm{~mm}$ deeper than the thickest boundary layer found for uniform stirring at the same rate). For the latter ranges, the computer control of the rod motion was programmed to pause the rods at their maximum depth for the time that they would otherwise have taken to traverse the tank, as indicated in the Fig. 1 inset. Thus, the return time, or intermittency, of the stirring is identical to that in the uniform stirring cases. This procedure ensures that the same diffusivity (and the same turbulence intermittency) is applied to the upper region in both cases. Stirring rates in all runs are chosen to be sufficiently large that additional momentum from the finite volume flux of the saline source is negligible (Stewart et al. 2012). Thus, $\kappa / \kappa_{m}>10^{2}$, where $\kappa$ is the measured turbulent diffusivity for salt and the smallest turbulent diffusivity is $4.76 \times 10^{-7} \mathrm{~m}^{2} \mathrm{~s}^{-1}$ (Table 1 ). The turbulent viscosity and effective Prandtl number are unknown for the stirring, which is not designed to produce a full inertial turbulence spectrum. The dynamically relevant Rayleigh number $\mathrm{Ra}_{\mathrm{E}}$ is estimated using the turbulent diffusivity and assuming the turbulent Prandtl number $\nu / \kappa \approx 1$, where $\nu$ is the effective viscosity. Thus, Rayleigh numbers $\mathrm{Ra}_{\mathrm{E}}=B L^{3} / \kappa^{2} \nu$ up to $O\left(10^{14}\right)$ are achieved.

The measurements focused on density (rather than the velocity field) as the most accurate means of characterizing the system. Density is measured using three parallel techniques, ensuring accuracy over the entire field. Vertical profiles of conductivity are taken near the end of the tank farthest from the plume $(x / L=0.86)$ and converted to profiles of density through a previously determined calibration function. A check of the accuracy of the salinity profiles is made by frequently 
withdrawing $2-\mathrm{mL}$ water samples from middepth $(z / D=-0.5)$ and measuring their densities with an Anton Paar digital density meter. These samples provide a time record in themselves and offer the most reliable indication of whether or not a flow had reached its longtime equilibrium state. The conductivity profiles provide detail on the vertical variation of density along with temporal changes, and the direct density measurements are used to correct small secular drifts in the conductivitydensity calibration.

The density field is also measured using digital imaging of transmitted light partially absorbed by trace concentrations of dye, following a similar method to that outlined in Holford and Dalziel (1996) and Allgayer and Hunt (2012). Blue food dye is added to the reservoir feeding the saline source. The dye "tracks" the salt through the system (as molecular diffusion played no significant role in any part of the circulation); the concentration evolves in the same way as the salinity and reaches an eventual equilibrium distribution. Digital images are taken looking through the tank against a uniformly illuminated background (provided by an electrofluorescent sheet placed behind the tank). The images record the attenuation in transmitted light intensity, from which the density of the solution can be determined; the maximum dye concentration indicates the maximum (source) density and the minimum concentration corresponds to freshwater. The light absorption technique offers horizontal averaging (along the path of a light ray) of the density field. The measurements have a high spatial resolution and are typically further averaged in $x, z$, and time (over small time windows), in contrast to the instantaneous, point measurements of density or profiles of conductivity. The method provides records of the approach to equilibrium that closely match those from density and conductivity. All three techniques are used in each run, with density samples sometimes restricted to early and late stages of an equilibration.

Most of the experiments (Table 1) begin with a homogeneous tank of salt solution (runs labeled with "a") with a density less than that expected in the equilibrium state, thus ensuring that the saline source produces a plume that falls to the bottom at all times. The surface fluxes and internal stirring are suddenly imposed and the approach to equilibrium monitored. In four cases the final state is then used as the initial condition for the next run (labeled with "b"), in which the imposed stirring is changed instantaneously and the flow allowed to readjust to a new equilibrium state. The change in imposed stirring is one of the following: 1) from one stirring rate through the full depth (a uniform diffusivity) to another,
2) from uniform stirring to upper region stirring with the same diffusivity, or 3) from one upper stirring rate to another. For most of the adjustments (runs labeled with "b") that begin from an equilibrium state with stirring, the diffusivity is decreased, as the expected response is for the boundary layer to become thinner, leading in turn to an increase in the plume density and continued penetration of the plume to the bottom. The change implies a net density flux into the working volume and the interior becoming denser. A change in the mixing regime in these four experiments, beginning from the equilibrium state established by a previous mixing regime, show most explicitly the effects of the differing mixing regimes. Whether the initial condition is a homogenous tank to which a given buoyancy flux and mixing regime are abruptly imposed, or an equilibrated circulation, all changes in forcing are designed to be abrupt so as not to introduce any externally imposed time scales.

In contrast to the relatively simple adjustments following a decrease of the mixing rate, an increase of the diffusivity causes the boundary layer to thicken, the plume to freshen, and a net salt flux out of the working volume. The evolution then involves a transitional period during which the plume penetrates only partway through the depth and forms a shallow convecting cell. The depth of convection slowly increases through penetrative convection until the plume again reaches the bottom, as previously studied in the thermally forced case (Rossby 1998; Mullarney et al. 2004; Griffiths et al. 2013), indicating that additional mechanisms are involved in the transient flow. Adjustment to an increase in diffusivity for stirring only in the upper region is therefore not considered, as the focus here is on the dynamics that also govern the equilibrium circulation and on the relative roles of mixing in the upper and interior regions.

The adjustment in the boundary layer is established rapidly, within $45 \mathrm{~min}$ of the forcing being applied. Over $10-20 \mathrm{~h}$ the densities asymptotically approach a final profile, in which the density at each depth is constant apart from minor fluctuations attributable to internal variability in the flow or small fluctuations in external variables (such as room temperature and source flow rate). Although we originally aimed to utilize only the adjustments between two slightly different convecting states given by two stirring regimes, the results show that the transient behavior was independent of the initial condition of either a homogeneous or fully convecting system. The overall duration of runs is constrained to $\sim 40 \mathrm{~h}$ (using close to the saline reservoir capacity of $400 \mathrm{~L}$ ), which limits observation of more than one adjustment between two convective states. Hence, we 
make full use of the data recorded during the initial adjustment.

\section{b. Experimental results}

If the interior is assumed to be only weakly stratified and hence effectively well mixed, then mixing in the surface boundary layer is of prime importance for the global circulation. A simple analysis of the buoyancy budget under these assumptions (Griffiths et al. 2013) predicts that normalized interior density evolves as an exponential throughout the adjustment process, eventually approaching the long-time asymptotic value. For the case of an imposed buoyancy flux (relevant to the salinity forcing in the present experiments),

$$
\frac{\rho(t)-\rho_{f}}{\rho_{i}-\rho_{f}}=e^{-t / \tau}, \quad \tau=h D / \beta \kappa,
$$

where $\tau$ is the decay time scale; $\rho(t)$ is the density of the interior that evolves in time; $\rho_{i}$ and $\rho_{f}$ are, respectively, the initial and final equilibrium densities of the interior; and $\beta$ is a constant of $O(1)$. Direct numerical simulations imply $\beta=1.4$; hence, $\tau=0.71 h D / \kappa$ (Griffiths et al. 2013). As the freshwater surface boundary condition is applied over the whole upper boundary, the time scale $\tau$ is a factor of 2 smaller than the previous thermally forced experiments, in which heating and cooling were each applied over one-half the surface area. The prediction [(1)] requires only that freshwater transport into the working volume through the surface (the freshwater interface) is governed by mixing within the boundary layer and that there is no substantial change in the small interior density gradient during an adjustment from one equilibrium state to another, as is the case when the diffusivity is decreased and interior density increases. Thus, the theory assumes that the ventilation time for the entire volume to pass through the boundary layer,

$$
t_{V}=A D / \psi
$$

where $A$ is the surface area and $\psi$ is the total transport, is small compared with $\tau$ given by (1), so that the interior gradients remain small (the experiments lie well within this regime and it will be seen in section 4 that the ocean model does not). For the case of applied flux boundary conditions and using scaling from Mullarney et al. (2004), the normalized ventilation time constant can be written as $\kappa t_{V} / D^{2} \sim(L / D) \mathrm{Ra}_{\mathrm{E}}^{-1 / 6}$ (Griffiths et al. 2013). Note also that a different exponential adjustment time scale is predicted for the case of imposed surface temperature or density boundary conditions, where buoyancy transport from the surface is governed by convection in a mixed layer that feeds into deep sinking near the boundary of the basin. In this case the time scale is simply (2) and, using scaling from Rossby (1965), the normalized time constant becomes $\kappa t_{V} / D^{2} \sim$ $(L / D) \mathrm{Ra}^{-1 / 5}$, where $\mathrm{Ra}=\Delta b L^{3} /(\nu \kappa)$ is the Rayleigh number for imposed specific buoyancy $\Delta b$.

Experimental results are summarized in Table 1. The water density $\rho$ is normalized as $\left(\rho-\rho_{0}\right) / \Delta \rho$. As a key indicator of behavior, we use the interior density at middepth $\rho^{*}$ and the corresponding normalized form $\left(\rho^{*}-\rho_{0}\right) / \Delta \rho$. The top-to-bottom density difference is $\left(\bar{\rho}_{\text {bot }}-\bar{\rho}_{\text {top }}\right) / \Delta \rho \approx\left(\rho^{*}-\rho_{0}\right) / \Delta \rho$ because the horizontal average of the density at the top of the working volume is $\bar{\rho}_{\text {top }} \approx \rho_{0}$, and as the interior vertical density gradient is in all cases small compared with that across the boundary layer, the middepth density serves as a close surrogate for the horizontally averaged bottom density $\bar{\rho}_{\text {bot }}$.

Provided that the mixing in the surface layer remains unchanged between two experiments, the same adjustment behavior is observed. This is shown in Fig. 2a where the final (equilibrium state) density profiles in runs $1 \mathrm{a}$ and $5 \mathrm{a}$ (the density profiles on the left) are essentially identical: these two runs have the same diffusivity in the upper region but in run 1a the interior diffusivity is the same as that in the upper region, whereas in run 5a there is no stirring in the interior. The remaining profiles correspond to the evolution of runs $1 \mathrm{~b}$ and $5 \mathrm{~b}$ and show that when the mixing rate is decreased by an order of magnitude, the flows evolve in a nearly identical manner. They approach the same new equilibrium stratification (the density profiles on the right). The temporal evolution of the interior densities for these runs is shown in Fig. 2b (against elapsed time normalized by external variables alone). The evolution is remarkably close to exponential, as predicted by (1) and observed in previous thermally driven overturning experiments (Griffiths et al. 2013), and the time constant $\tau$ is not dependent on the level of mixing in the interior. A small difference in boundary layer thickness (Table 1; discussed further below) contributes to the uncertainty of the predicted time scale, which corresponds to $\kappa \tau / D^{2}=0.71 h / D$.

For comparison with (1), the $e$-folding time scale for each run is renormalized to the form $\kappa \tau / h D$, using the measured values of $\tau$ and $h$ (Table 1 ) and plotted against the diffusivity applied by the stirring rods (Fig. 3). Here, the diffusivity is normalized by the constant molecular value (as the simplest possible reference and without implication that the ratio has significance). In Fig. 3, the normalized time scales are compared with and without the presence of interior mixing, and we conclude that the time scales show no significant dependence on the level of interior stirring across the accessible range of applied diffusivity. The time scales are also independent of 

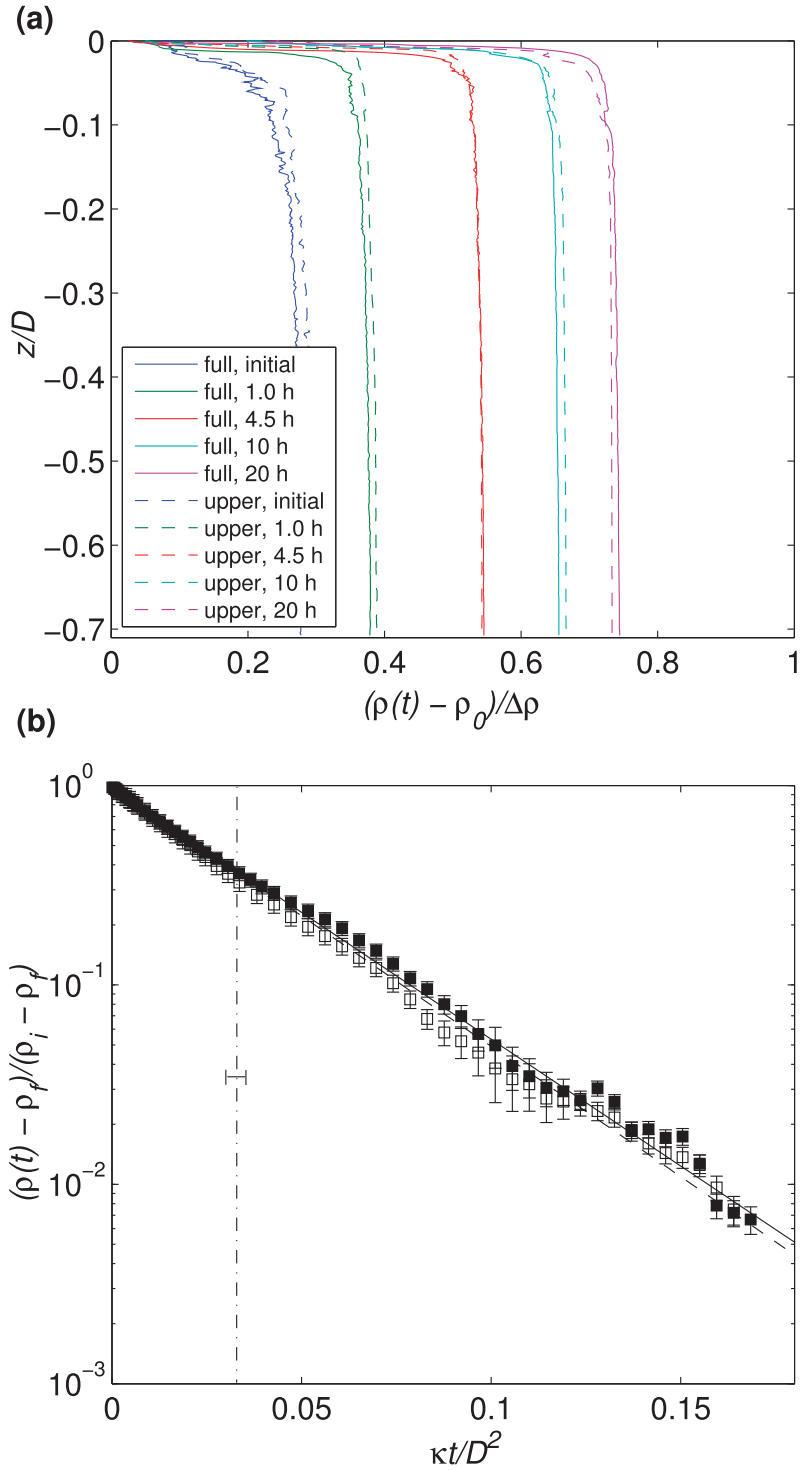

FIG. 2. Evolution of density after a decrease in vertical diffusivity for uniform, full-depth stirring (solid lines, run 1b) and upper stirring only (broken lines, run 5b). (a) Vertical profiles (from left to right) of the initial equilibrium $(t=0), 1.0,4.5,10$, and $20 \mathrm{~h}$ after the change in stirring. In both runs, density increased with time and the last profiles (right) represent the final equilibrium states. (b) Normalized density at middepth for full-depth $\mathbf{\square}$ and upper region $\square$ stirring. Time is normalized by external variables. The initial states (with the exception of a minor difference in $\rho_{i}$ ) and the imposed diffusivities (in the upper region) were identical in the two runs, the only difference being the depth of mixing. Uncertainties (derived largely from fluctuations in the final equilibrium state) are indicated, and straight lines are the best-fit exponentials of the form in (1). Systematic uncertainties in $\kappa$ are not shown (see Table 1). The vertical broken line in (b) indicates the predicted value of the $e$-folding time $\tau=0.71 h D / \kappa[(1)]$ using the measured $h$, along with the uncertainty on that predicted value corresponding to the difference in $h$ between the two runs. In run $1 \mathrm{~b}, \rho_{i}=1004.96 \mathrm{~kg} \mathrm{~m}^{-3}$ and $\rho_{f}=1016.02 \mathrm{~kg} \mathrm{~m}^{-3}$, and in run $5 \mathrm{~b} \rho_{i}=1005.25 \mathrm{~kg} \mathrm{~m}^{-3}$ and $\rho_{f}=1015.42 \mathrm{~kg} \mathrm{~m}^{-3}$.

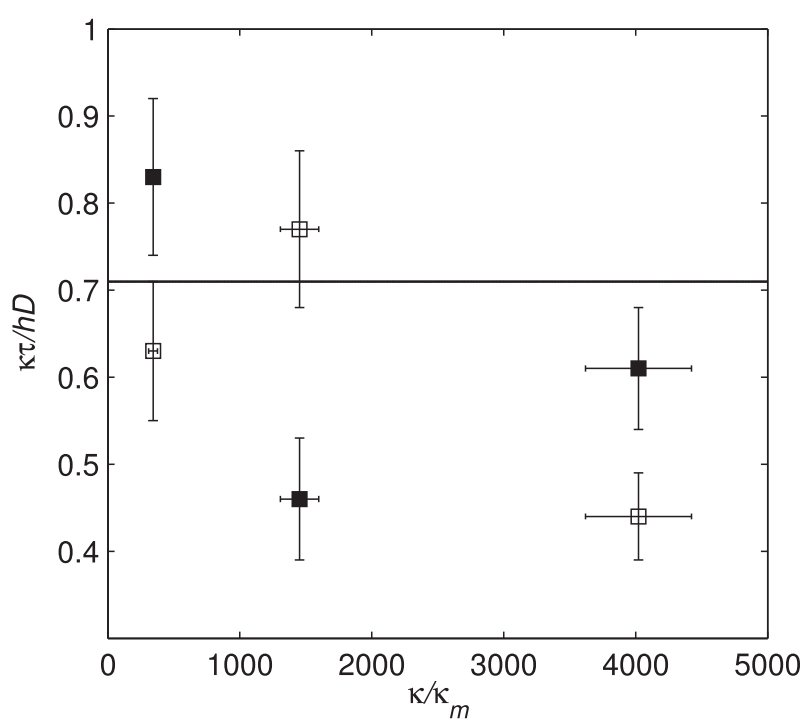

FIG. 3. Normalized exponential time scales for flow adjustments plotted against the imposed turbulent diffusivity. The time scales are normalized by $h D / \kappa$, where $h$ is the measured boundary layer depth; diffusivity is normalized by the molecular diffusion coefficient for density. Symbols indicate full-depth $\mathbf{a}$ and upper region $\square$ stirring; error estimates are based on uncertainties in $h$ and $\tau$. Only runs in which the plume sank to the bottom at all times are included. The line is the model prediction $[(1)] \tau=0.71 \mathrm{hD} / \kappa$ (as also shown in Fig. 2b).

whether convection is initiated from a homogeneous tank or from a fully developed convection state and whether the diffusivity is decreased while maintaining the same depth of stirring or is changed from uniform to shallow stirring. The average value $\kappa \tau / h D \simeq 0.6 \pm 0.1$ is in agreement with the predicted value 0.71 , supporting the hypothesis that the circulation is governed by diffusion within the boundary layer. Remaining unexplained is a trend to smaller normalized time scales at larger diffusivities.

For completeness, the stirring rate was also increased by an order of magnitude. The flow adjustment involved a period of shallow circulation, and the $e$-folding time scale was up to a factor of 2 larger (Table 1; run $2 b)$. Increasing the diffusivity leads to a greater uptake of freshwater into the boundary layer, which in turn makes the plume more buoyant, causing it to cease sinking to the bottom and intrude into the interior below the boundary layer. This partial-depth stirring drives a shallow circulation for a period of several hours until full-depth overturning is reestablished. Density profiles (Fig. 4) show a strong density gradient extending through much of the domain and particularly through the shallow circulation cell at early stages in the adjustment. In run 3, the stirring was applied to a homogenous tank of initially greater density than the 


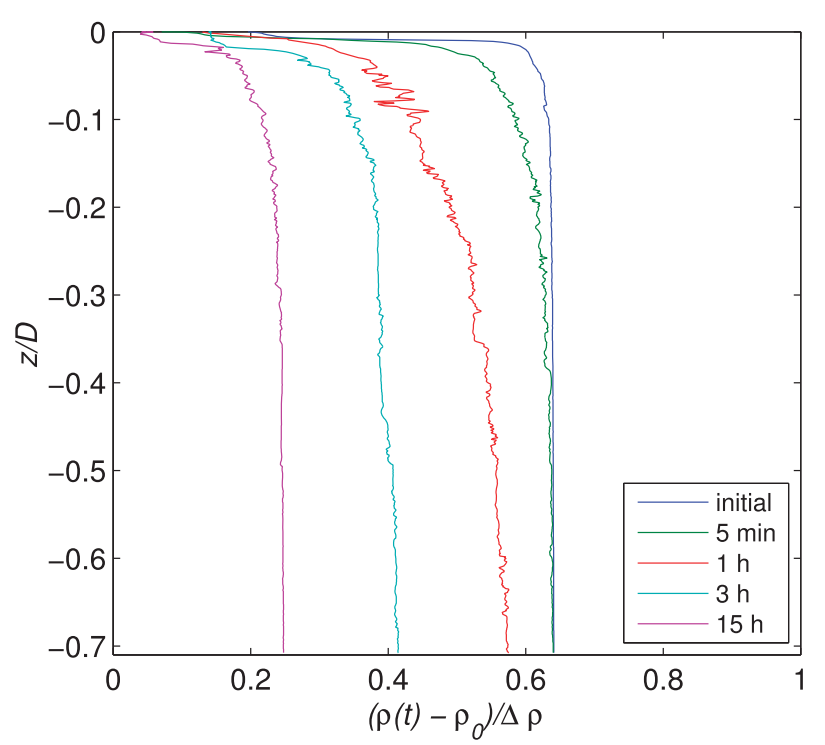

FIG. 4. An example of the adjustment of stratification after an increase in diffusivity imposed uniformly through the full depth (run 2b), the reverse of the change shown in Fig. 2. Sequence is right to left as the density decreases; profiles are taken in the initial equilibrium state and at $5 \mathrm{~min}$ and 1,3 , and $15 \mathrm{~h}$ (the final equilibrium) after the change in $\kappa$. There is greater stratification during the temporary phase of shallow circulation.

equilibrium state; hence, the system also had to undergo a decrease in density and followed a similar adjustment path as run $2 b$. The equilibration of runs $2 b$ and 3 is not well described by the simple exponential [(1)], a behavior also shared with transient thermal horizontal convection adjusting under a net input of stabilizing buoyancy (an overall warming under the top surface forcing; Griffiths et al. 2013). For these reasons, this case is less useful in providing information about the dynamics of the equilibrium circulation. However, we note that the adjustment time is again of order $h D / \kappa$, and therefore a factor $D / h$ smaller than the time for turbulent diffusion through the full depth. The final equilibrium state also matches the equivalent case approaching equilibrium via an increase in density (cf. run $1 \mathrm{a}$ with run $2 \mathrm{~b}$ and run $1 \mathrm{~b}$ with run 3 ).

In the equilibrium state the interior density and topto-bottom density difference, plotted in Fig. 5a, are insensitive to whether stirring (at a given rate) is applied through the full depth or only within the shallow upper region ( $\kappa$ being the upper diffusivity in the latter case). The density differences are also consistent with scaling theory (Hughes et al. 2007), which for a similar, nonrotating, rectangular basin and endwall line plume gives

$$
\left(\bar{\rho}_{\text {bot }}-\bar{\rho}_{\text {top }}\right) / \Delta \rho=2.46\left(\kappa g^{\prime 2} L / B^{5 / 3}\right)^{-1 / 2} .
$$

The scaling theory assumes a uniform diffusivity throughout the depth, and the present equilibrium
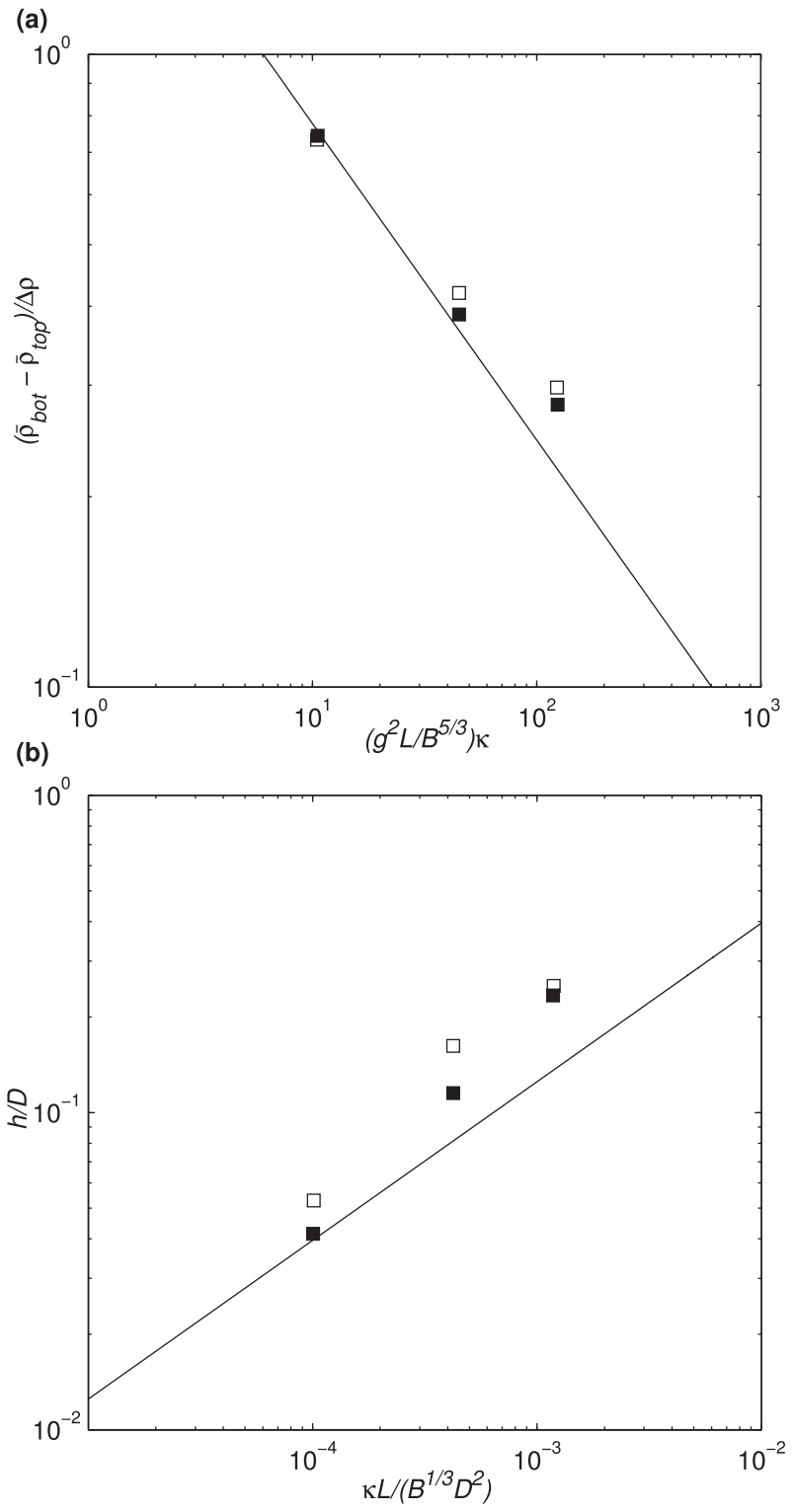

FIG. 5. A comparison of equilibrium states from runs $1 \mathrm{a}$ and $1 \mathrm{~b}$, $4 \mathrm{a}$ and $4 \mathrm{~b}$, and $5 \mathrm{a}$ and $5 \mathrm{~b}$ with full-depth $\boldsymbol{\square}$ or upper region $\square$ stirring: (a) top-to-bottom density difference $\approx\left(\rho^{*}-\rho_{0}\right) / \Delta \rho$; (b) normalized boundary layer depth $h / D$. Both quantities are plotted as functions of (dimensionless) diffusivity for three mixing rates that give $\kappa=4.76 \times 10^{-7}, 2.02 \times 10^{-6}$, and $5.59 \times 10^{-6} \mathrm{~m}^{2} \mathrm{~s}^{-1}$ (left to right) (other quantities in the abscissa varied relatively little). Lines show the theoretical predictions (3) and (4) taken from Hughes et al. (2007) for the line plume case; the lines have slope $-1 / 2$ and $1 / 2$, respectively.

results for this case are similar to the previous experimental results of Stewart et al. (2012). The vertical density profiles for run $4 \mathrm{~b}$ in Fig. 6 (the middiffusivity case in Fig. 5a) demonstrate that when the depth of stirring is suddenly confined to the upper region (after convection was first established with uniform full-depth 


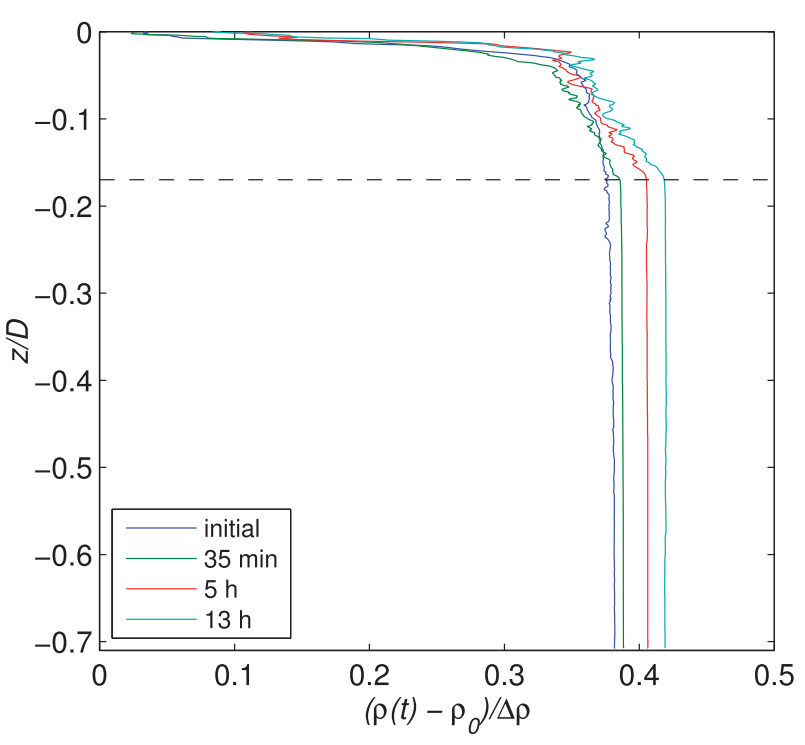

FIG. 6. Vertical density profiles show that very little adjustment of stratification took place when full-depth stirring (in an overturning previously brought to equilibrium) was suddenly changed to upper region stirring with the same diffusivity (run $4 \mathrm{~b}$ in Table 1 ): (left to right) profiles (increasing density) show the initial equilibrium convection and at $35 \mathrm{~min}$ and 5 and $13 \mathrm{~h}$ (the final equilibrium) after the depth of stirring was changed. Broken line is the depth of upper stirring for this run $(90 \mathrm{~mm}$ below freshwater interface).

stirring), only a minor increase of the interior density occurs. The slightly smaller top-to-bottom density differences (maximum $3 \%$ of $\Delta \rho$ ) for full-depth stirring (compared to upper stirring) can possibly be attributed to increased lateral mixing and entrainment into the plume owing to the application of stirring through a greater range of depths. Alternatively, the elevated level of interior mixing when the stirring is full depth could result in a slightly smaller density difference.

The equilibrium boundary layer thickness $h$ (Table 1 and plotted in Fig. 5b) is defined as that containing 95\% of the top-to-bottom density difference in the flow at large times. For uniform stirring, our trend in $h$ is consistent with previous experiments (Stewart et al. 2012) and scaling theory (Hughes et al. 2007):

$$
h / D=3.95(\kappa L)^{1 / 2} /\left(B^{1 / 6} D\right),
$$

although the results for the two larger diffusivities indicate a boundary layer somewhat thicker than predicted. For the largest mixing rate (runs 1a and 5a) the two values of $h$ are not significantly different. When stirring is confined to the upper region (cf. run $1 \mathrm{~b}$ with run $5 \mathrm{~b}$ in Table 1 and run $4 \mathrm{a}$ with run $4 \mathrm{~b}$ ), $h$ is $30 \%-40 \%$ larger than for full-depth stirring. This increase in boundary layer thickness for run $4 \mathrm{~b}$ shows a change in profile shape (Fig. 6), with the upper part of the boundary layer little changed from that under full-depth stirring, while a region of relatively small density gradient formed throughout the depth of the upper stirring. The reason for these differences in $h$ remains unclear. However, the differences are secondary when compared to the variation of $h$ with the stirring rate. We conclude that an imposed turbulent diffusivity gives rise to similar interior density and stratification in the long-time equilibrium state, independent of whether or not the stirring is present in the interior. This is consistent with the transient results that show that the adjustment time scales are set by buoyancy uptake into, or rejection from, the boundary layer and not by turbulent mixing in the interior.

\section{Ocean model}

\section{a. Model design}

The ocean model MITgcm (Marshall et al. 1997a,b) is used to solve for circulation in an idealized ocean extending from $70^{\circ} \mathrm{S}$ to $70^{\circ} \mathrm{N}, 40^{\circ}$ wide, and $4000 \mathrm{~m}$ deep with sloping boundaries and a reentrant channel in the southern part of the domain, which is the geometry used by Shakespeare and Hogg (2012) and pictured in Fig. 7a. Flow is forced by realistic meridional distributions of zonal surface wind stress and relaxed to a prescribed sea surface buoyancy profile on a time scale of 20 days [Figs. 7b,c; buoyancy is defined as $b \equiv g\left(\rho_{0}-\rho\right) / \rho_{0}=$ $g \alpha T$, where $T$ is model temperature in ${ }^{\circ} \mathrm{C}, \alpha$ is a constant thermal expansion coefficient, $\rho_{0}=1025 \mathrm{~kg} \mathrm{~m}^{-3}$, and there is no salinity forcing]. The flow is solved under the Boussinesq and hydrostatic approximations. The model resolution is $1 / 4^{\circ}$ on a Mercator grid, chosen to partially represent eddy processes in the Southern Ocean, with the vertical resolution (36 levels) ranging from $10 \mathrm{~m}$ at the surface to $200 \mathrm{~m}$ at depth.

The depth dependence of the vertical diffusion coefficient $K$ is prescribed as $K=K_{A}+\left(K_{U}-K_{A}\right) e^{-(z / \gamma)^{2}}$, with an abyssal (or interior) value $K_{A}$ applied throughout most of the domain and a larger value $K_{U}$ in the upper ocean, as summarized in Table 2 and Fig. $7 \mathrm{~d}$, with the diffusivity change occurring over a length scale of $\gamma=150 \mathrm{~m}$. While the terms "abyssal" and "upper" diffusivity are used to describe the idealized model, the upper region does not necessarily correspond to the ocean thermocline. The two vertical diffusivities are varied independently while all other conditions are fixed. We note that the rate of uptake or rejection of buoyancy from the surface is set in part by $K_{U}$ and in part by a range of other processes such as the relaxation boundary condition, the convection parameterization, and the circulation. That is to say, the specified model 
(a)

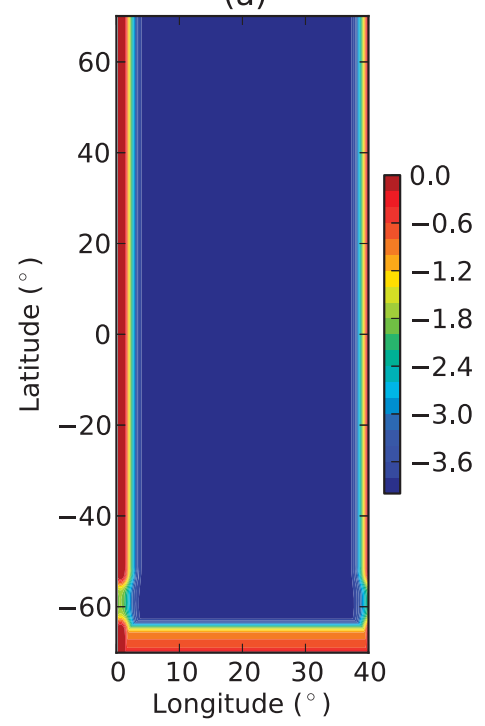

(b)

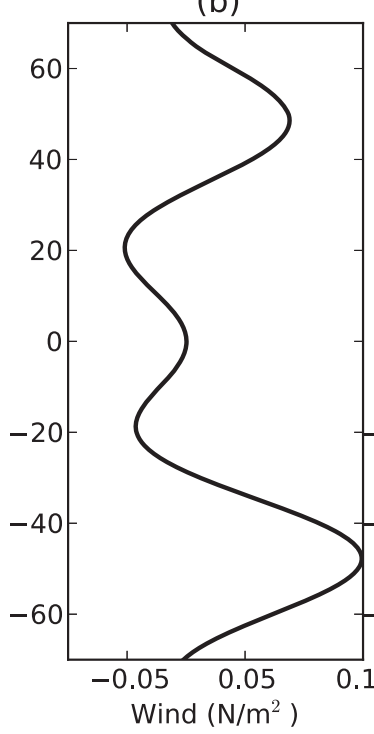

(c)

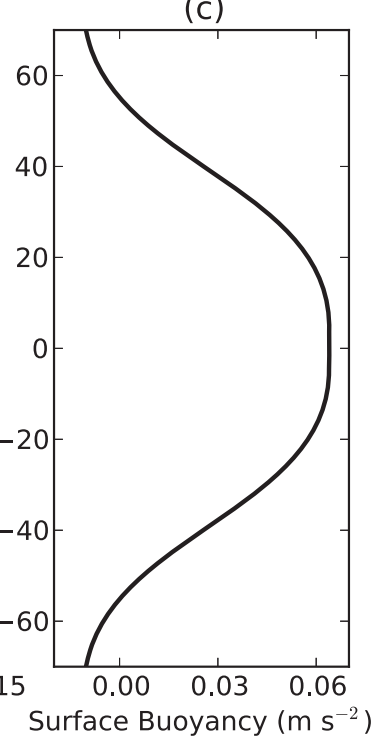

(d)

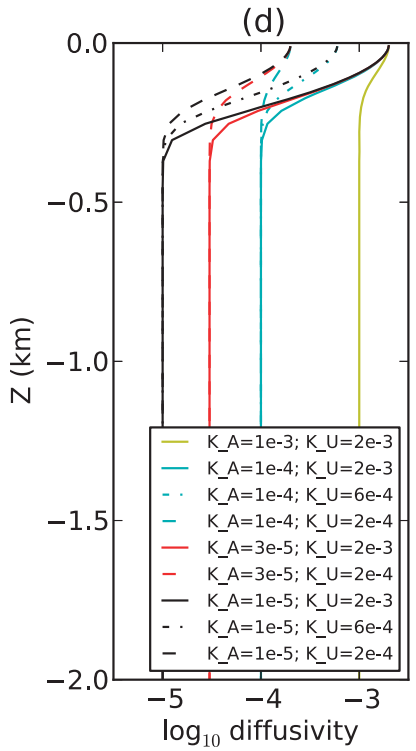

FIG. 7. (a) The model ocean bathymetry $(\mathrm{km})$, (b) zonal surface wind stress, (c) freshwater surface buoyancy flux, and (d) the profile of vertical diffusivity $\left(\mathrm{m}^{2} \mathrm{~s}^{-1}\right)$ for nine different model simulations.

diffusivity $K_{U}$ does not play the same role as an elevated turbulent diffusivity at the surface in the laboratory experiment. Hence, the model runs can be interpreted as varying the abyssal diffusivity considerably, while variations of $K_{U}$ are only partly responsible for controlling the buoyancy uptake. The combination of wind stress and a reentrant channel in the southern part of the domain naturally leads to an upper and abyssal cell, and the boundary conditions permit the relative areas of cooling and heating to adjust.

We carried out nine simulations initiated from a homogeneous ocean of buoyancy $b=0.02 \mathrm{~m} \mathrm{~s}^{-2}$, chosen such that the ocean proceeds to cool and the sinking leg of the overturning is at all times more dense than the abyssal water. Overturning therefore remains full depth throughout the adjustment and the approach to the long-term thermal equilibrium. Additionally, a "coldstart" simulation (denoted $2 \mathrm{~A}^{\prime}$ ) is conducted with the same parameters as $2 \mathrm{~A}$ except the initial buoyancy $b=-0.01 \mathrm{~ms}^{-2}$, in which case the solution adjusts through a period of shallow circulation. The length of each simulation is $1600 \mathrm{yr}$, except for the run with a very large abyssal diffusivity (case 1 in Table $2 ; K_{A}=1 \times$ $10^{-3} \mathrm{~m}^{2} \mathrm{~s}^{-1}$ ) that came to the equilibrium state quickly and was thus only run for $1000 \mathrm{yr}$.

\section{b. Model results}

As the simulations approach equilibrium the circulation is characterized by the familiar upper-ocean western boundary currents and midlatitude gyres, with a

TABLE 2. Ocean model runs with the diffusivity in the upper and abyssal regions, respectively, $K_{U}$ and $K_{A}$; the $e$-folding time scale in the upper and lower overturning cells $\tau_{U}$ and $\tau_{A}$; the time scale based on diffusion through the full depth $D^{2} / K_{A}$; and the time scale based on advection through the upper and lower cells $A h_{U} / \psi_{U}$ and $A\left(D-h_{U}\right) / \psi_{A}$.

\begin{tabular}{|c|c|c|c|c|c|c|c|}
\hline Run & $K_{U}\left(\mathrm{~m}^{2} \mathrm{~s}^{-1}\right)$ & $K_{A}\left(\mathrm{~m}^{2} \mathrm{~s}^{-1}\right)$ & $\tau_{U}(\mathrm{yr})$ & $A h_{U} / \psi_{U}(\mathrm{yr})$ & $\tau_{A}(\mathrm{yr})$ & $D^{2} / K_{A}(\mathrm{yr})$ & $A\left(D-h_{U}\right) / \psi_{A}(\mathrm{yr})$ \\
\hline 1 & $2 \times 10^{-3}$ & $1 \times 10^{-3}$ & 75 & 60 & 85 & 500 & 120 \\
\hline $2 \mathrm{~A}$ & $2 \times 10^{-3}$ & $1 \times 10^{-4}$ & 140 & 120 & 340 & 5000 & 500 \\
\hline $2 \mathrm{~A}^{\prime}$ & $2 \times 10^{-3}$ & $1 \times 10^{-4}$ & 55 & 120 & 620 & 5000 & 550 \\
\hline $2 B$ & $6 \times 10^{-4}$ & $1 \times 10^{-4}$ & 140 & 125 & 340 & 5000 & 550 \\
\hline $2 \mathrm{C}$ & $2 \times 10^{-4}$ & $1 \times 10^{-4}$ & 140 & 130 & 340 & 5000 & 550 \\
\hline $3 \mathrm{~A}$ & $2 \times 10^{-3}$ & $3 \times 10^{-5}$ & 190 & 150 & 450 & 17000 & 670 \\
\hline $3 \mathrm{C}$ & $2 \times 10^{-4}$ & $3 \times 10^{-5}$ & 190 & 175 & 430 & 17000 & 760 \\
\hline $4 \mathrm{~A}$ & $2 \times 10^{-3}$ & $1 \times 10^{-5}$ & 205 & 170 & 450 & 50000 & 780 \\
\hline $4 B$ & $6 \times 10^{-4}$ & $1 \times 10^{-5}$ & 205 & 190 & 430 & 50000 & 840 \\
\hline $4 C$ & $2 \times 10^{-4}$ & $1 \times 10^{-5}$ & 210 & 210 & 430 & 50000 & 870 \\
\hline
\end{tabular}



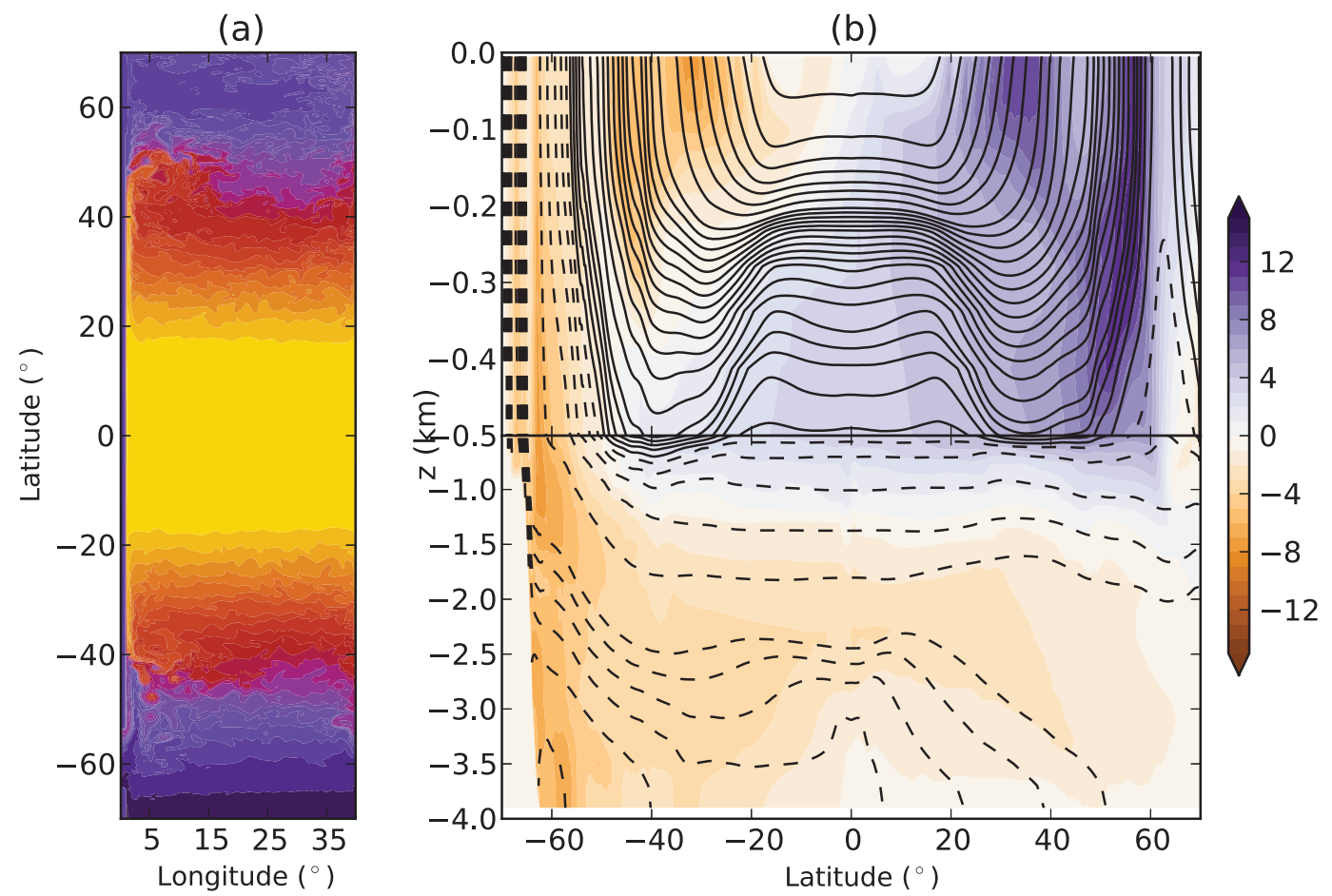

FIG. 8. (a) Surface buoyancy distribution (range from -0.01 to $0.06 \mathrm{~m} \mathrm{~s}^{-2}$ ) and (b) overturning transport [colorscale indicates transport in Sverdrups $\left(\mathrm{Sv} ; 1 \mathrm{~Sv} \equiv 10^{6} \mathrm{~m}^{3} \mathrm{~s}^{-1}\right)$; positive is clockwise in this vertical plane] and buoyancy distribution (black contours; negative values are indicated by broken lines) for the near-equilibrium state in case 4A.

strong Antarctic Circumpolar Current (see the surface buoyancy snapshot in Fig. 8a). In the tropics, a strongly stratified thermocline overlies a weakly stratified interior, with the base of the thermocline at approximately $300 \mathrm{~m}$ in the case of enhanced upper mixing (indicated by buoyancy contours in Fig. 8b), while the polar thermocline stratification is considerably weaker. The abyssal buoyancy frequency is relatively uniform, and the difference in horizontally averaged densities between the base of the thermocline and the bottom, $\Delta b=$ $g\left(\rho_{\text {base }}-\rho_{\text {bot }}\right) / \rho_{0} \sim 0.005 \mathrm{~m} \mathrm{~s}^{-2}$ (or at $1500 \mathrm{~m}, \Delta b=$ $\left.g\left(\rho_{1500}-\rho_{\text {bot }}\right) / \rho_{0} \sim 0.002 \mathrm{~m} \mathrm{~s}^{-2}\right)$, is comparable to that observed in the ocean. The residual overturning transport for case 4A, calculated on density surfaces and remapped to $z$ space, is indicated by the colors in Fig. 8b. This circulation shows a two-cell structure, with a relatively shallow upper cell outcropping in the north and intruding into the Southern Hemisphere at depths of 500 to $1500 \mathrm{~m}$, above a deeper (and weaker) abyssal cell.

The model solutions (except the cold-start case $2 \mathrm{~A}^{\prime}$ ) indicate a largely exponential adjustment of the horizontally averaged buoyancy at any chosen depth toward a large time value (Fig. 9, where, for comparison with the laboratory experiments, we plot the buoyancy differences $\Delta b$ between the surface and middepth and between the surface and the bottom, less the corresponding large time value $\Delta b_{\text {eq }}$, and normalized by the overall imposed surface buoyancy difference $\Delta b_{0}$ ). The behavior has many similarities to the present laboratory experiments (Fig. 2b) and to that observed previously in both laboratory experiments and DNS of thermal horizontal convection (Griffiths et al. 2013). In particular, the middepth ocean responds quickly to the imposed forcing, the buoyancy adjusting exponentially in the first $500-800 \mathrm{yr}$ before approaching a state that is in equilibrium with the boundary conditions (Fig. 9a, with $e$-folding time scales $\tau_{U}$, noted in Table 2). Natural fluctuations become more obvious on the logarithmic scale of Fig. 9a as the model solutions approach equilibrium, again as in thermal horizontal convection, after more than $97 \%$ of the adjustment is complete. The surface-to-bottom buoyancy difference (Fig. 9b) shows a departure from the simple exponential, which too is similar to that found in horizontal convection, when the adjustment is so large as to be comparable to the driving surface buoyancy difference.

In the cold-start run $2 \mathrm{~A}^{\prime}$, the upper cell adjustment occurs on a faster time scale $\tau_{U}$ than in run $2 \mathrm{~A}$; middepth buoyancy approaches equilibrium within $300 \mathrm{yr}$. As expected, the abyssal ocean (Fig. 9b, with $e$-folding time scales $\tau_{A}$ in Table 2) takes a much longer time to react and at least twice the time to equilibrate. This 
(a) [Surface - Mid-Depth] Buoyancy

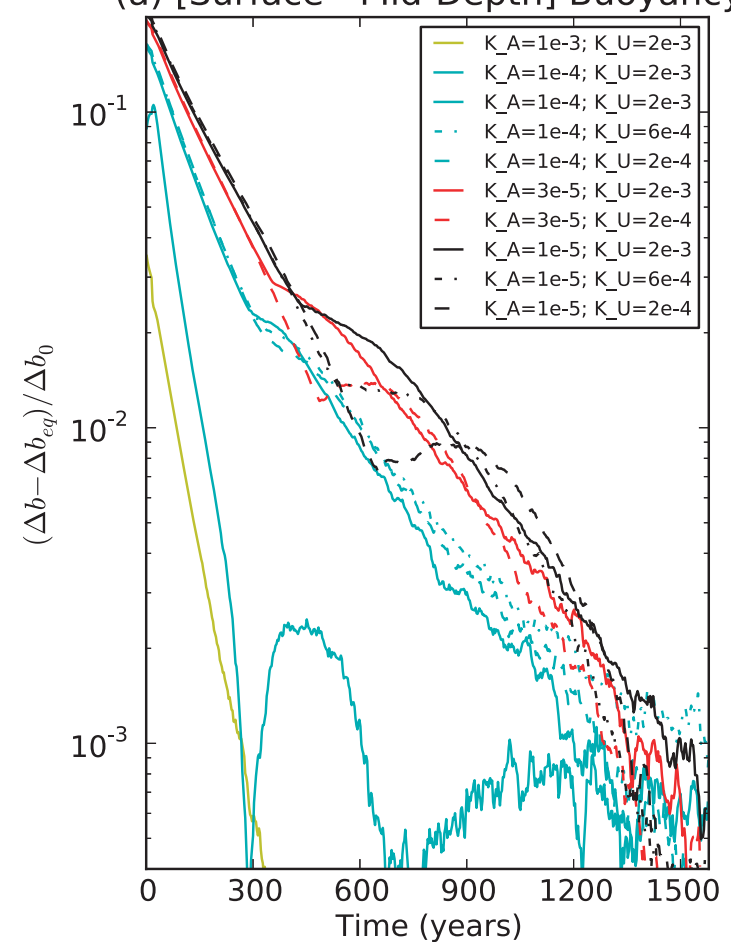

(b) [Surface - Abyssal] buoyancy

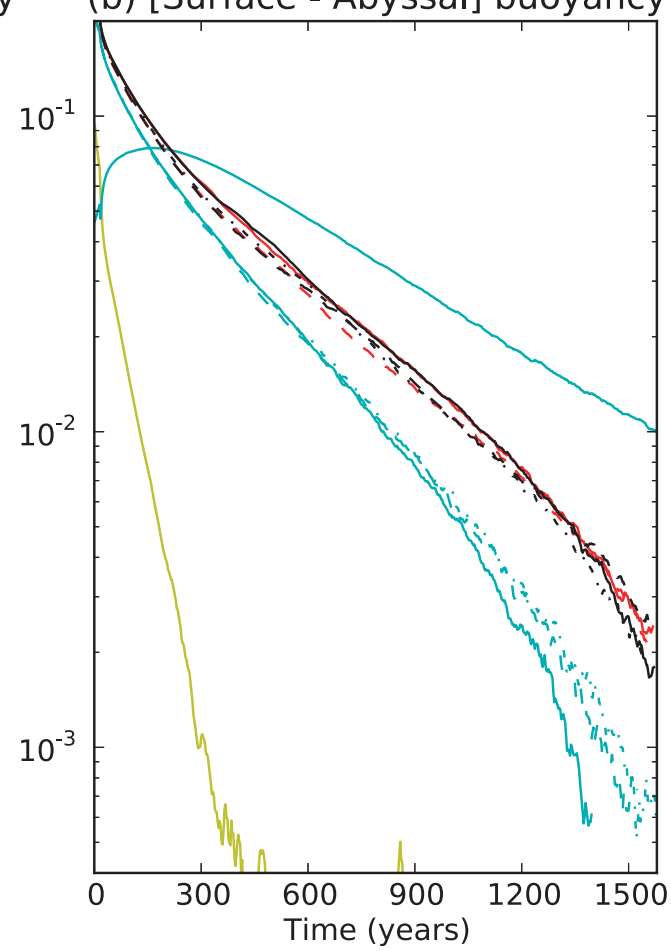

FIG. 9. Semilog plot of time series of (a) normalized buoyancy difference between the surface and middepth $(\sim 1500 \mathrm{~m})$; (b) normalized buoyancy difference between the surface and the bottom grid cell. Line colors show different abyssal diffusivities $K_{A}$; line styles show different upper diffusivities $K_{U}$.

asymmetry to net warming and net cooling can be explained by the time taken to erode the stable stratification in the abyss, which in these simulations is essentially a diffusive process. In the present laboratory experiments (section $2 b$ ), the asymmetry (in the time scale) was similarly a factor of 2 but involved penetrative convection that could break up the stable stratification, and in the thermally forced experiments of Griffiths et al. (2013), there was a wider range of asymmetry depending on the kind of boundary conditions and the changes in boundary forcing to which the flow had to adjust. One difference in the present solutions is an abyssal density that proceeds to increase throughout the flow adjustment and that does not reach an asymptotic density at the largest times solved, as indicated by the mismatch between the cold-start case and the equivalent warm-start case (solid cyan lines).

The effects on the transient adjustment of the buoyancy field of different abyssal diffusivity can be seen by comparing the solutions shown by solid lines (or comparing the dashed lines) in Fig. 9. There is a strong dependence of middepth buoyancy upon abyssal diffusivity; in particular, the rate of adjustment in the first 500-800 yr is governed to first order by the value of $K_{A}$. After the bulk of the adjustment has taken place there is a break in the curve, the timing of which is a function of upperocean diffusivity $K_{U}$ (shown by lines of different styles). The exponential time scales of adjustment are summarized in Table 2 and Fig. 10. The abyssal buoyancy adjustment time scale $\tau_{A}$ shows a dependence upon $K_{A}$ and is independent of $K_{U}$ when $K_{A}$ is larger than $3 \times$ $10^{-5} \mathrm{~m}^{2} \mathrm{~s}^{-1}$, whereas this time scale has no dependence on $K_{A}$ when $K_{A}$ is smaller. At the same time, the middepth buoyancy adjusts on the time scale $\tau_{U}$, which is effectively independent of the upper diffusivity value across the range examined but is weakly dependent on the abyssal diffusivity (approximately as $K_{A}^{-1 / 4}$ ).

The meridional transport is zonally integrated on density surfaces, and the maximum streamfunction values are used to characterize the transport in each of the upper and lower overturning cells. As a result of larger intrinsic variability in near-equilibrium conditions, these metrics are plotted in linear coordinates (Fig. 11). The evolution of transport in the upper cell (Fig. 11a) is insensitive to both upper and lower diffusivity (except for the extreme case of run 1), and the circulation adjusts toward equilibrium much faster than the buoyancy.

The solutions indicate that the buoyancy at the end of each run is only weakly dependent on both upper and 


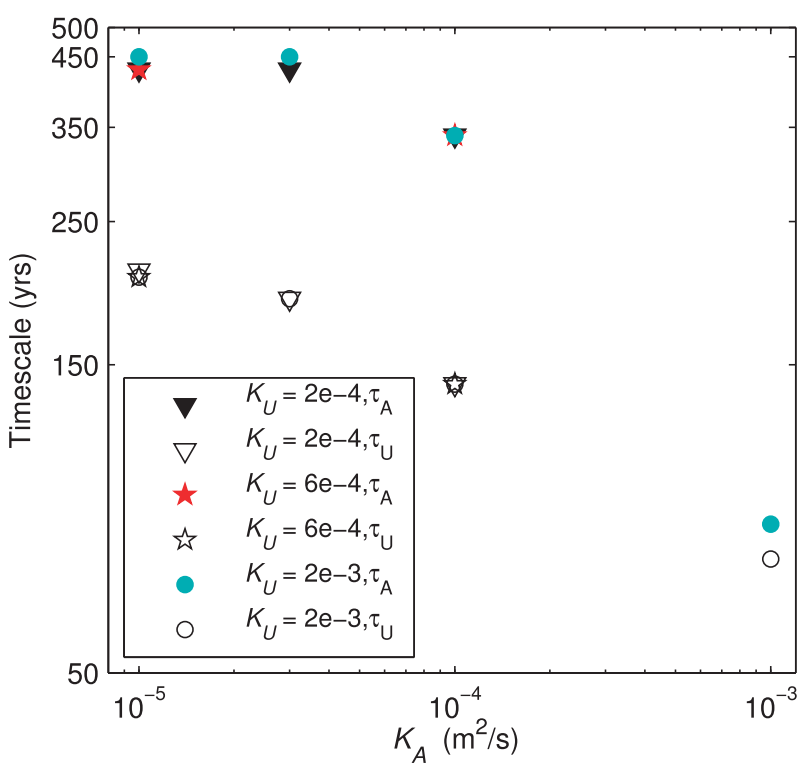

FIG. 10. Adjustments from Table 2 where $\tau_{U}$ is upper cell time scale and $\tau_{A}$ is abyssal cell time scale.

lower diffusivity (Figs. 12a,b). Here, we assume that the ends of the runs are representative of the final equilibrium states, which are approached exponentially slowly. Over the three values of $K_{A}$ the power-law dependence is weaker than $\Delta b \sim K_{A}^{-0.06}$ for surface to middepth and $\Delta b \sim K_{A}^{-0.02}$ for surface to bottom over the range of $K_{A}$ considered. The $K_{U}$ dependence is even weaker than this. The equilibrium overturning transport (Figs. 12c,d), on the other hand, is more sensitive to diffusivities. Transport in both the upper and lower cells also shows a power-law variation with $K_{A}$ over the full range but with a constant factor comparable to the exponent and hence a significantly stronger dependence at $K_{A}>3 \times 10^{-5} \mathrm{~m}^{2} \mathrm{~s}^{-1}$. There the dependence is roughly $\psi \sim K_{A}^{1 / 4}$ and stronger than the dependence on the upper diffusivity under these conditions. At smaller $K_{A}$ the dependence reduces to approximately $K_{A}^{0.1} K_{U}^{0.1}$. Hence, the roles of mixing in the model change as the solutions approach close to equilibrium. We focus on the transient adjustment.

The model time scales are compared with the exponential time constant [(2)], which is expected to apply if the adjustment is limited by the rate at which the ocean volume can be ventilated in the boundary layer. We note that there is a different key transport mechanism (Griffiths et al. 2013); adjustments for imposed flux conditions (as represented in the imposed flux experiments reported in this paper) are governed by vertical diffusion in the thermocline, whereas adjustments under imposed buoyancy conditions (closer to the conditions used in the present ocean model runs) are expected to be governed by convection over the cooled and locally unstable regions of the surface. Given the complexities of the ocean model circulation, we allow a modification of (2) for the depth and volume of each overturning cell, so that the time constant for the upper cell becomes

$$
\tau_{U}=\frac{\text { volume }}{\psi}=\frac{A h_{U}}{\psi_{U}},
$$

where $A$ is again the surface area, $h_{U}$ is an estimate of the depth of the upper cell ( $1500 \mathrm{~m}$ is used here), and $\psi_{U}$ is the volume transport through the boundary layer, for which we use the equilibrium value. In a similar vein, for the lower cell we replace $h_{U}$ in (5) with $D-h_{U}$ and the transport with $\psi_{A}$. The resulting predicated advective time scales are listed in Table 2 and prove a good firstorder approximation of the adjustment of the stratification in the present ocean model results, with a numerical prefactor for the scaling in (5) of approximately 1.14 for the upper cell and 0.60 for the lower cell adjustments. Although the time scales in Fig. 10 are plotted against abyssal diffusivity, the relationship between these variables is indirect; the evolution is governed by the advection time scale, which is influenced by the abyssal diffusivity (and very weakly by the upper diffusivity) via the overturning transport (Figs. 12c,d). This mechanism allows a relatively weak influence of diffusivity on the adjustment time scale, without requiring diffusivity alone to explicitly govern the evolution. The importance of convective transport from the surface under these boundary conditions (which effectively impose the rate of buoyancy uptake), in place of diffusion through the thermocline, explains the absence of a dependence on the upper diffusivity $K_{U}$.

\section{Discussion}

The laboratory experiment and ocean model results together highlight the effects of the different kinds of boundary conditions; the former has been shown to result in adjustment time scales controlled by diffusion within the boundary layer and the latter is controlled by convectively unstable regions of the boundary layer. Both regimes rely on the adjustment of interior (or abyssal) buoyancy via transport through the upper boundary layer, and the adjustment times therefore depend on the depth of overturning. Neither time scale depends explicitly on the abyssal mixing rate (although there is an indirect dependence of the model time scale on abyssal diffusivity via the volume transport term). Although it is possible that Kelvin and Rossby wave propagation may influence the ocean model equilibration for up to several hundred years after the start of the run (Huang et al. 2000), our model results show that the 

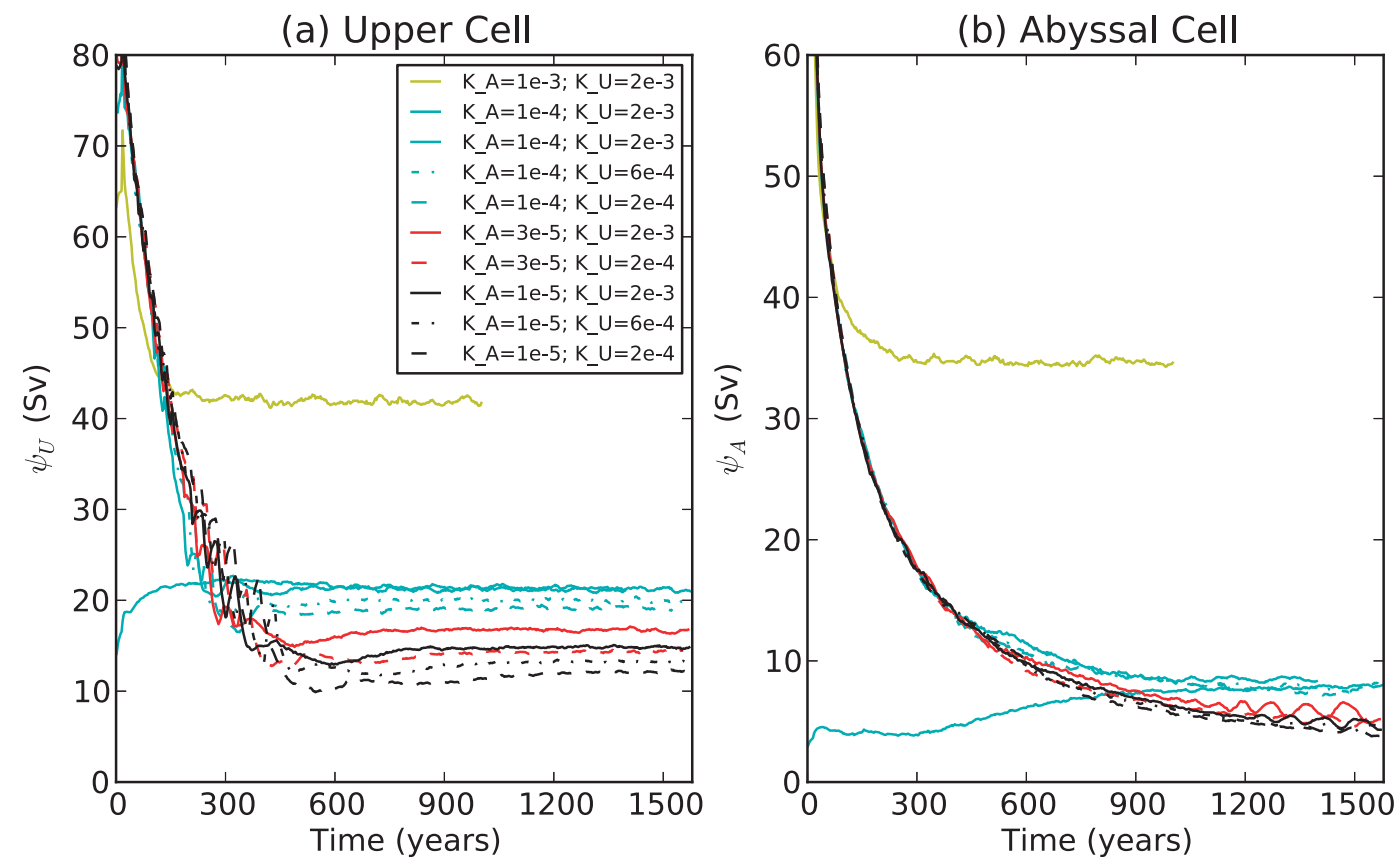

FIG. 11. Time series of (a) the upper overturning circulation magnitude and (b) the abyssal overturning for all 10 experiments. Line styles and colors are as in Fig. 9.

exponential time scale is largely consistent with the advection time. Another key observation is that, as was the case in the laboratory experiments, the $e$-folding time scales from the ocean model are much shorter than the times for turbulent diffusion through the ocean depth (5000-50000 yr for the abyssal diffusivities used in the model cases).

The insensitivity of the adjustment time scale to changes of interior vertical diffusivity, in both the laboratory experiments and the ocean model (at low $K_{A}$ ), is consistent with the large Peclet number characterizing the interior circulation. The interior Peclet number can be defined as $\mathrm{PE}_{\mathrm{int}}=w_{\mathrm{int}} D / \kappa$, based on the interior vertical velocity $w_{\text {int }}$ and interior diffusivity $\kappa$ (or $K_{A}$ ). To calculate the Peclet number for the laboratory experiments, we use equilibrium state vertical velocities of $w_{\text {int }}$ from the related study by Stewart et al. (2012); thus, $\mathrm{PE}_{\text {int }} \sim 50$ (using $w_{\text {int }} \sim 10^{-4} \mathrm{~m} \mathrm{~s}^{-1}, D \approx 0.5 \mathrm{~m}$, and $\left.\kappa \sim 10^{-6} \mathrm{~m}^{2} \mathrm{~s}^{-1}\right)$. In the numerical ocean model, $\mathrm{PE}_{\mathrm{int}} \sim 1$ in the abyssal cell requires $K_{A} \sim 1 \times 10^{-4} \mathrm{~m}^{2} \mathrm{~s}^{-1}$, and in the upper cell it requires $K_{U} \sim 2 \times 10^{-4} \mathrm{~m}^{2} \mathrm{~s}^{-1}$ (the surface area of the domain is $A \sim 10^{14} \mathrm{~m}^{2}$ ), which accords well with the transition from a weak to a strong dependence on diffusivity in the numerical results. In the ocean $\mathrm{PE}_{\text {int }} \sim 40$ (using $w_{\text {int }} \sim 10^{-7} \mathrm{~m} \mathrm{~s}^{-1}, D \approx$ $4000 \mathrm{~m}$, and $\kappa \sim 10^{-5} \mathrm{~m}^{2} \mathrm{~s}^{-1}$; Polzin et al. 1997; Lumpkin and Speer 2007; Whalen et al. 2012). Hence, for abyssal mixing to be of substantial importance in adjustments of the ocean overturning, the average diffusivity in the interior (given this vertical velocity, based on estimates of the actual overturning rate) would need to approach $4 \times 10^{-4} \mathrm{~m}^{2} \mathrm{~s}^{-1}$. Thus, we conclude that if the oceans are not in equilibrium, they may be dominated by advective transport to the extent that vertical mixing is important only in the thermocline or surface mixed layer. The key role of buoyancy uptake into the surface layers highlights the significance of the surface mixed layer and, particularly, the deep winter convective layer at high latitudes in the North Atlantic (Kara et al. 2003).

In evaluating the model results it should be remembered that although the adjustment rates of nonequilibrium circulation are predicted to be governed by convective mixing in the unstable boundary layer, the model includes convection only through a "convective adjustment" parameterization. The diffusion coefficient required to parameterize the removal of heat from the model domain by this process is not well constrained. As mixing in horizontal convection is almost all in the boundary layer (Gayen et al. 2013) and the energy consumed by mixing must be equal to the available potential energy input by surface buoyancy fluxes (Hughes et al. 2009), it may also be problematic that small-scale convection in the surface mixed layer is not resolved by ocean models and is instead parameterized as a vertical diffusivity.

Regarding equilibrium states, the ocean model results differ from those of the laboratory experiments. The modeled stratification in both the upper and lower cells 


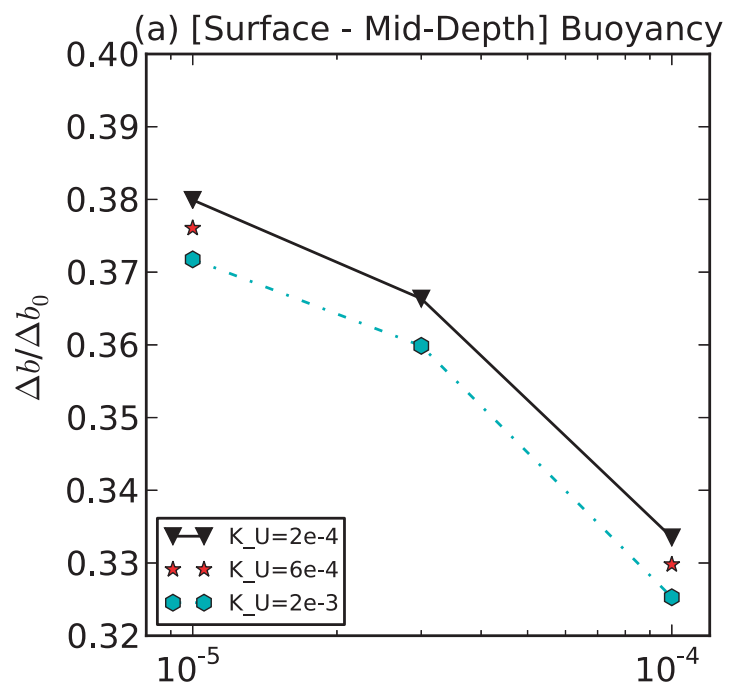

(b) [Surface - Abyssal] buoyancy
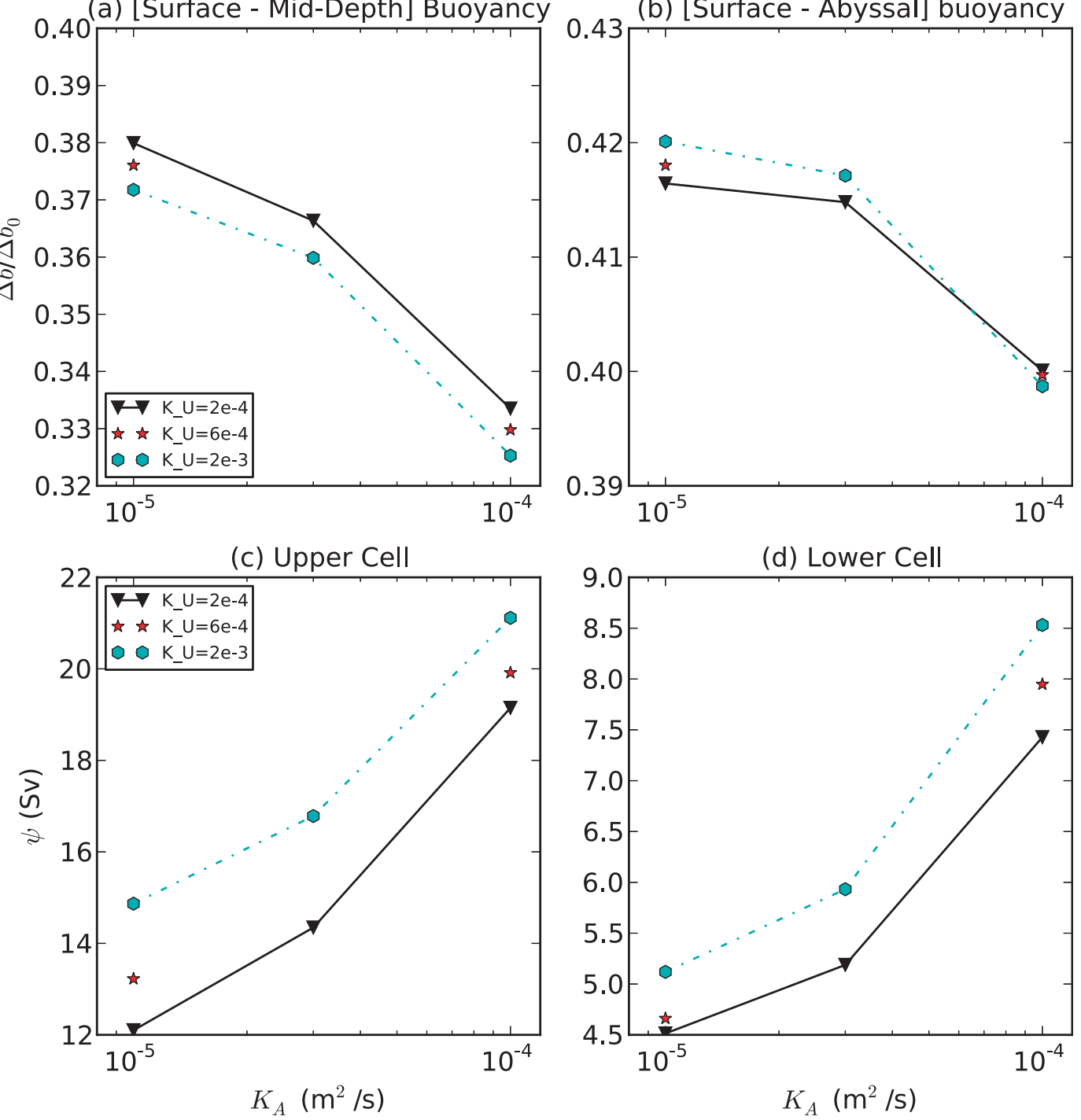

FIG. 12. Large time values of (a) the surface to middepth buoyancy difference, (b) the surface to abyssal buoyancy difference, (c) the transport in the upper overturning cell, and (d) the transport in the lower overturning cell between $45^{\circ}$ and $55^{\circ} \mathrm{S}$. All values are averaged over the final $100 \mathrm{yr}$ of model simulations and are taken to indicate nearequilibrium values. Note that axes are semilogarithmic.

is only very weakly dependent on mixing rate, rather than the strong dependence (on upper diffusivity to the $-1 / 2$ power; Fig. $5 a$ ) in the laboratory system. The equilibrium overturning transport in the ocean model is dependent on upper diffusivity as $K_{U}^{0.1}$ and on abyssal diffusivity broadly as $K_{A}^{0.2}$, with a weaker dependence at small $K_{A}$. In the laboratory model, measurements of transport for uniform diffusivity through the full depth [reported in Stewart et al. (2012)] indicate that the transport changes as $\kappa^{1 / 4}$. However, these differences are not surprising because, as argued above, the upper diffusivity in the model is unlikely to characterize the removal of heat from the model domain.
The model results are consistent with those of Nikurashin and Vallis $(2011,2012)$, where at low diffusivity, the stratification and overturning were not determined by the diapycnal diffusivity but were instead governed by wind stress and eddy effects in the circumpolar channel. The transition to this wind stressdominated regime was found to occur at a diffusivity of $10^{-5}-10^{-4} \mathrm{~m}^{2} \mathrm{~s}^{-1}$ (Nikurashin and Vallis 2011). Hence, the increased sensitivity to upper mixing in the present ocean model might be a result of the abyssal diffusivity being small enough to place the model in the weakly diffusive regime, whereas the upper diffusivity (which in all cases was greater than or equal to $2 \times 10^{-4} \mathrm{~m}^{2} \mathrm{~s}^{-1}$ ) 
was sufficiently large that it influenced the circulation. It seems likely that the present model results for $K_{A}=$ $10^{-4} \mathrm{~m}^{2} \mathrm{~s}^{-1}$ place our idealized ocean model close to a strongly diffusive regime, but only after the circulation approaches sufficiently close to an equilibrium state. At that stage the advective transports, initially dominant, become less important. On the other hand, at the smallest abyssal diffusivity considered $\left(K_{A}=\right.$ $10^{-5} \mathrm{~m}^{2} \mathrm{~s}^{-1}$; the smallest accessible in such a numerical model without spurious numerical diffusion playing a significant role), even the nearly equilibrated abyssal solution is in a largely nondiffusive, advectiondominated regime. Wolfe and Cessi (2011) also find that wind stress across the Southern Ocean, coupled with mixing at isopycnals outcropping in the North Atlantic, is required for a pole-to-pole quasi-adiabatic circulation to be supported; otherwise, only weak diffusive cells are present. Such a reliance on surface mixing is consistent with both the laboratory experiment and ocean model results reported here, which indicate that the region of buoyancy uptake at the surface governs the adjustment and circulation.

\section{Conclusions}

We have studied the adjustment of the meridional overturning circulation in a laboratory experiment and an ocean model. In the laboratory experiment, the mixing rate in the near surface was varied independently to that of the interior, while the ocean model had separate diapycnal diffusivities prescribed in the abyss and in the upper ocean. This approach has yielded insight into the relative roles of surface and abyssal processes for mass transport and equilibration of the overturning circulation. The adjustment time scales in both the laboratory experiment and ocean model are largely independent of the abyssal diffusivity (unless the model is in a strongly diffusive regime, which requires an abyssal diffusivity $K_{A} \gtrsim 3 \times 10^{-5} \mathrm{~m}^{2} \mathrm{~s}^{-1}$ ).

The complementary laboratory experiments and ocean model, with different boundary conditions and enormously different levels of complexity, help us to clarify the physics governing the adjustment of the circulation and consistently show that the adjustment time scales are not set by the abyssal mixing. The experiments show adjustment time scales that are dependent upon the near-surface mixing rate, whereas the model runs have adjustment time scales set by advective ventilation rates. The result that different processes set the adjustment time scales in the experiments and ocean model is explained by the different manner in which buoyancy is supplied or rejected at the surface; in our experiments the bulk buoyancy in the circulation changes only via diffusive transfer at the surface, whereas restoring boundary conditions in the ocean model extract buoyancy and cool the bulk circulation via convection in the regions of destabilizing surface buoyancy flux. The latter convection is also a parameterized process that is not strongly dependent on the upper-ocean diffusivity.

The results indicate that equilibration of the global ocean (with a weak to moderately diffusive abyss, $K_{A} \lesssim$ $3 \times 10^{-5} \mathrm{~m}^{2} \mathrm{~s}^{-1}$ in the model) involves water mass transformation almost entirely confined to the surface, with advection throughout the volume as the dominant transport process. Adjustments are likely to be governed by the ventilation time based on the ocean volume and the rate of overturning-typically an exponential time scale on the order of hundreds of years and much less than the time scale based on turbulent diffusion through the depth.

Acknowledgments. We thank T. Beasley for construction of equipment and laboratory assistance. The research was supported by Australian Research Council Grants DP1094542 and DP12010274. GOH and AMH were supported by ARC Future Fellowships FT10010086 and FT120100842, respectively. This research was undertaken on the NCI National Facility in Canberra, Australia, which is supported by the Australian Commonwealth Government.

\section{REFERENCES}

Allgayer, D. M., and G. R. Hunt, 2012: On the application of the light-attenuation technique as a tool for non-intrusive buoyancy measurements. Exp. Therm. Fluid Sci., 38, 257-261, doi:10.1016/j.expthermflusci.2011.10.009.

Bryan, F., 1987: Parameter sensitivity of primitive equation ocean general circulation models. J. Phys. Oceanogr., 17, 970-985, doi:10.1175/1520-0485(1987)017<0970:PSOPEO>2.0.CO;2.

Cummins, P. F., G. Holloway, and A. E. Gargett, 1990: Sensitivity of the GFDL ocean general circulation model to a parameterization of vertical diffusion. J. Phys. Oceanogr., 20, 817-830, doi:10.1175/1520-0485(1990)020<0817:SOTGOG > 2.0.CO;2.

de Verdière, A. C., 1988: Buoyancy driven planetary flows. J. Mar. Res., 46, 215-265, doi:10.1357/002224088785113667.

Ferrari, R., and D. Ferreira, 2011: What processes drive the ocean heat transport? Ocean Modell., 38, 171-186, doi:10.1016/ j.ocemod.2011.02.013.

Gayen, B., R. W. Griffiths, G. O. Hughes, and J. A. Saenz, 2013: Energetics of horizontal convection. J. Fluid Mech., 716, R10, doi:10.1017/jfm.2012.592.

Griffiths, R. W., G. O. Hughes, and B. Gayen, 2013: Horizontal convection dynamics: Insights from transient adjustment. J. Fluid Mech., 726, 559-595, doi:10.1017/jfm.2013.244.

Holford, J. M., and S. B. Dalziel, 1996: Measurements of layer depth during baroclinic instability in a two-layer flow. Appl. Sci. Res., 56, 191-207, doi:10.1007/BF02249381.

Huang, R. X., M. A. Cane, N. Naik, and P. Goodman, 2000: Global adjustment of the thermocline in response to deepwater 
formation. Geophys. Res. Lett., 27, 759-762, doi:10.1029/ 1999 GL002365.

Hughes, G. O., and R. W. Griffiths, 2006: A simple convective model of the global overturning circulation, including effects of entrainment into sinking regions. Ocean Modell., 12, 46-79, doi:10.1016/j.ocemod.2005.04.001.

J. C. Mullarney, and W. H. Peterson, 2007: A theoretical model for horizontal convection at high Rayleigh number. J. Fluid Mech., 581, 251-276, doi:10.1017/S0022112007005630.

_ A. M. Hogg, and R. W. Griffiths, 2009: Available potential energy and irreversible mixing in the meridional overturning circulation. J. Phys. Oceanogr., 39, 3130-3146, doi:10.1175/ 2009JPO4162.1.

Jayne, S., 2009: The impact of abyssal mixing parameterizations in an ocean general circulation model. J. Phys. Oceanogr., 39, 1756-1775, doi:10.1175/2009JPO4085.1.

Johnson, H. L., and D. P. Marshall, 2002: A theory for the surface Atlantic response to thermohaline variability. J. Phys. Oceanogr., 32, 1121-1132, doi:10.1175/1520-0485(2002)032<1121: ATFTSA $>2.0 . C O ; 2$.

Kara, A. B., P. A. Rochford, and H. E. Hurlburt, 2003: Mixed layer depth variability over the global ocean. J. Geophys. Res., 108, 3079, doi:10.1029/2000JC000736.

Kawase, M., 1987: Establishment of deep ocean circulation driven by deep-water production. J. Phys. Oceanogr., 17, 2294-2317, doi:10.1175/1520-0485(1987)017<2294:EODOCD>2.0.CO;2.

Lumpkin, R., and K. Speer, 2007: Global ocean meridional overturning. J. Phys. Oceanogr., 37, 2550-2562, doi:10.1175/JPO3130.1.

Marotzke, J., and B. Klinger, 2000: The dynamics of equatorially asymmetric thermohaline circulations. J. Phys. Oceanogr., 30, 955970, doi:10.1175/1520-0485(2000)030<0955:TDOEAT >2.0.CO;2.

Marshall, J., and K. Speer, 2012: Closure of the meridional overturning circulation through Southern Ocean upwelling. Nat. Geosci., 5, 171-180, doi:10.1038/ngeo1391.

_, A. Adcroft, C. Hill, L. Perelman, and C. Heisey, 1997a: A finite-volume, incompressible Navier Stokes model for studies of the ocean on parallel computers. J. Geophys. Res., 102, 5753-5766, doi:10.1029/96JC02775.

, C. Hill, L. Perelman, and A. Adcroft, 1997b: Hydrostatic, quasi-hydrostatic, and nonhydrostatic ocean modeling. J. Geophys. Res., 102, 5733-5752, doi:10.1029/96JC02776.

Mignot, J., A. Leverlmann, and A. Griesel, 2006: A decomposition of the Atlantic meridional overturning circulation into physical components using its sensitivity to vertical diffusivity. J. Phys. Oceanogr., 36, 636-650, doi:10.1175/JPO2891.1.

Mullarney, J. C., R. W. Griffiths, and G. O. Hughes, 2004: Convection driven by differential heating at a horizontal boundary. J. Fluid Mech., 516, 181-209, doi:10.1017/S0022112004000485.

Munk, W., 1966: Abyssal recipes. Deep-Sea Res. Oceanogr. Abstr., 13, 707-730, doi:10.1016/0011-7471(66)90602-4.

_ and wind mixing. Deep-Sea Res. I, 45, 1977-2010, doi:10.1016/ S0967-0637(98)00070-3.

Nikurashin, M., and G. Vallis, 2011: A theory of deep stratification and overturning circulation in the ocean. J. Phys. Oceanogr., 41, 485-502, doi:10.1175/2010JPO4529.1.

- , and - 2012: A theory of the interhemispheric meridional overturning circulation and associated stratification. J. Phys. Oceanogr., 42, 1652-1667, doi:10.1175/JPO-D-11-0189.1.
—, and R. Ferrari, 2013: Overturning circulation driven by breaking internal waves in the deep ocean. Geophys. Res. Lett., 40, 3133-3137, doi:10.1002/grl.50542.

Polzin, K. L., J. M. Toole, J. R. Ledwell, and R. W. Schmitt, 1997: Spatial variability of turbulent mixing in the abyssal ocean. Science, 276, 93-96, doi:10.1126/science.276.5309.93.

Robinson, A., and H. Stommel, 1959: The oceanic thermocline and the associated thermohaline circulation. Tellus, 11A, 295-308, doi:10.1111/j.2153-3490.1959.tb00035.x.

Rossby, H. T., 1965: On thermal convection driven by non-uniform heating from below: An experimental study. Deep-Sea Res. Oceanogr. Abstr., 12, 9-16, doi:10.1016/0011-7471(65)91336-7.

- 1998: Numerical experiments with a fluid non-uniformly heated from below. Tellus, 50A, 242-257, doi:10.1034 j.1600-0870.1998.t01-1-00006.x.

Saenko, O. A., and W. J. Merryfield, 2005: On the effect of topographically enhanced mixing on the global ocean circulation. J. Phys. Oceanogr., 35, 826-834, doi:10.1175/JPO2722.1.

Samelson, R. M., 1998: Large-scale circulation with locally enhanced vertical mixing. J. Phys. Oceanogr., 28, 712-726, doi:10.1175/1520-0485(1998)028<0712:LSCWLE>2.0.CO;2.

Scott, J. R., and J. Marotzke, 2002: The location of diapycnal mixing and the meridional overturning circulation. J. Phys. Oceanogr., 32, 3578-3595, doi:10.1175/1520-0485(2002)032<3578: TLODMA $>2.0 . \mathrm{CO} ; 2$.

Shakespeare, C. J., and A. M. Hogg, 2012: An analytical model of the response of the meridional overturning circulation to changes in wind and buoyancy forcing. J. Phys. Oceanogr., 42 1270-1287, doi:10.1175/JPO-D-11-0198.1.

Stewart, K., 2011: The effects of sills and mixing on the meridional overturning circulation. Ph.D. thesis, Australian National University, $113 \mathrm{pp}$.

_, G. O. Hughes, and R. W. Griffiths, 2012: The role of turbulent mixing in an overturning circulation maintained by surface buoyancy forcing. J. Phys. Oceanogr., 42, 1907-1922, doi:10.1175/ JPO-D-11-0242.1.

Toggweiler, J. R., and B. Samuels, 1998: On the oceans large-scale circulation near the limit of no vertical mixing. J. Phys. Oceanogr., 28, 1832-1852, doi:10.1175/1520-0485(1998)028<1832: OTOSLS $>2.0 . \mathrm{CO} ; 2$.

Welander, P., 1971: The thermocline problem. Philos. Trans. Roy. Soc. London, A270, 415-421, doi:10.1098/rsta.1971.0081.

Whalen, C. B., L. D. Talley, and J. A. MacKinnon, 2012: Spatial and temporal variability of global ocean mixing inferred from Argo profiles. Geophys. Res. Lett., 39, L18612, doi:10.1029/ 2012 GL053196.

Whitehead, J. A., and W. Wang, 2008: A laboratory model of vertical ocean circulation driven by mixing. J. Phys. Oceanogr., 38, 1091-1106, doi:10.1175/2007JPO3805.1.

Wolfe, C. L., and P. Cessi, 2011: The adiabatic pole-to-pole overturning circulation. J. Phys. Oceanogr., 41, 1795-1810, doi:10.1175/2011JPO4570.1.

Yang, J., 1999: A linkage between decadal climate variations in the Labrador Sea and the tropical Atlantic Ocean. Geophys. Res. Lett., 26, 1023-1026, doi:10.1029/1999GL900181.

Zhang, J., R. W. Schmitt, and R. X. Huang, 1999: The relative influence of diapycnal mixing and hydrologic forcing on the stability of the thermohaline circulation. J. Phys. Oceanogr., 29, 1096-1108, doi:10.1175/1520-0485(1999)029<1096:TRIODM>2.0.CO;2. 